\title{
Synthesis, antimicrobial and antiradical activity of (3-alkoxymethyl-4-hydroxyphenyl)propan-1-ones, intermediates of biologically active compounds and activity comparison with 3-(alkoxymethyl)-4-(alkylamino-2- hydroxypropoxyphenyl)alkanones type of beta blockers
}

\author{
${ }^{1}$ Department of Chemical Theory of Drugs, \\ Faculty of Pharmacy, Comenius University in Bratislava \\ 2Department of Cellular and Molecular Biology of Drugs, \\ Faculty of Pharmacy, Comenius University in Bratislava
}

Received 4 April, 2020, accepted 23 January, 2021

Abstract A homologous series of (3-alkoxymethyl-4-hydroxyphenyl)propan-1-ones was prepared by the reaction of (3-chloromethyl4-hydroxyphenyl)propan-1-ones with the corresponding alcohols (methanol - decan-1-ol, propan-2-ol, 2-methylpropan-1-ol, 3-methylbutan-1-ol, cyclopentanol, benzylalcohol) in the presence of sodium hydrogen carbonate. The composition of the synthesised compounds was elucidated by IR, UV and ${ }^{1} \mathrm{H}-\mathrm{NMR}$ and ${ }^{13} \mathrm{C}-\mathrm{NMR}$ spectra. Selected compounds were tested against human pathogens: gram-positive bacterium Staphylococcus aureus (CNCTC Mau 29/58), gram-negative bacterium Escherichia coli (CNCTC 377/79) and yeast Candida albicans (CCM 8186). Their antimicrobial activities were expressed as minimum inhibitory concentrations. Antioxidant activity was determined using DPPH and ABTS.+ methods. It could be shown that both biological activities, antimicrobial and antioxidant, were lower in comparison with the (2RS)-bis [3-(4-acetyl-2-propoxymethyl)phenoxy-2hydroxypropyl]isopropylammonium fumarate type of beta blockers.

Keywords substituted phenols - antimicrobial activity - antioxidant activity - antiradical activity

\section{INTRODUCTION}

The introduction of a phenolic group into drug molecules confers upon them a reactive functionality with acidic nature. Phenols can form chelates with metal ions (Hider et al., 2001; Fernandez et al., 2002) and are able to bind to basic functional groups of proteins, hence their broad spectrum of biological activities, such as antimicrobial (Taguri et al., 2004; Taguri et al., 2006; Cueva et al., 2010; Park et al., 2001) and antioxidative activity (Sroka \& Cisowski, 2003; Bendary et al., 2013).

The bioactivity of phenols and their toxicity are both influenced by the number of phenolic groups and their relative position (Calliste et al., 2001; Amouar et al., 2009; Kadoma et al., 2010).

Fujisawa (Fujisawa et al., 2004) studied antioxidative activity of 2-methoxy- and 2-tert-butylphenols having up to three substituents on the aromatic ring. It could be shown that antioxidative activity decreases as a result of exchange of the methoxymethyl group against a methyl group. Derivatives with a tert-butyl group exhibited marked increase in antioxidative activity.

Kadoma et al. (2008) and Kadoma et al. (2009) investigated, besides antioxidative properties, also the cytotoxicity of 2- or 2,6-tert-butylphenols and 2-methoxyphenols in several cancer cell lines. Both antioxidative and cytotoxic activities followed similar structure-activity relationship. The introduction of a tert-butyl group as well as dimerisation led to a distinct increase in cytotoxicity.

The structurally similar eugenol (2-methoxy-4-(prop-2-en-1yl)phenol) exhibits antibacterial (Devi et al., 2010), antifungal (Morcia et al., 2012; Abbaszadeh et al., 2014), antioxidant 
(Fujisawa et al., 2002), local anaesthetic (Park et al., 2001) and anti-depressant activities (Irie et al., 2004).

Herein, we report a two-stage synthesis of the target compounds starting from 4-hydroxyphenylpropan-1-one (Table 1 and Figure 1). In the first step, the electrophilic substitution reaction of 4-hydroxyphenylpropan-1one with paraformaldehyde and hydrochloric acid yields 1-[3-(chloromethyl)-4-hydroxy-phenyl]propan-1one. Nucleophilic substitution of this intermediate by corresponding alcohols leads to 1-[-3-(alkoxymethyl)-4hydroxyphenyl]propan-1-ones, where the substituent can be an aliphatic alkyl with chain length $C_{1}-C_{10^{\prime}}$ cyclopentyl and phenylmethyl (benzyl).

Many of these substances find application as intermediates in the synthesis of biologically active compounds of the aryloxyaminopropanol type with beta adrenoceptor blocking, antiarrhythmic and anticonvulsive activity (Čižmáriková et al., 1985; Čižmáriková et al., 1986; Čižmáriková et al., 2003).

\section{EXPERIMENTAL}

The melting points were determined using a Kofler micro hot stage and were quoted uncorrected. Elemental analysis was carried out on a FLASH 2000 (Thermo Scientific) analyser, and the results were within $0.3 \%$ of the theoretical values.

The purity of newly prepared compounds was assessed by TLC using Silufol" UV 254 (Merck) sheets with the mobile phase cyclohexane/ethyl acetate (8:2 v/v). UV spectra were recorded on the spectrometer Hewlett-Packard 8452 in methanol. IR spectra were measured using FTIR IMPACT 400 D (Nicolet) 6700. ${ }^{1} \mathrm{H}-\mathrm{NMR}$ were recorded on Varian Gemini 2000 spectrometer operating at 3,000 MHz for protons.

\section{Synthesis}

\section{(3-Chloromethyl-4-hydroxyphenyl)propan-1-one} (Čižmáriková et al., 1991)

To a sulfonation flask setup with mechanical stirring contact thermometer and powder funnel, $0.15 \mathrm{~mol}$ of 4-hydroxyphenylpropan-1-one (1a) and $90 \mathrm{~cm}^{3}$ of concentrated $\mathrm{HCl}$ were added. The temperature was subsequently maintained, the mixture was stirred and the reaction was allowed to proceed for $4.5 \mathrm{~h}$. Following the precipitation, the solid product was collected using suction filtration, washed with water and crystallised from benzene or ethyl acetate. M.p. $132-5^{\circ} \mathrm{C}$, yield $75 \%$ (da Re \& Verlichi, 1956) m.p. $133-6^{\circ} \mathrm{C}$, yield $57 \%$ ).

\section{(3-Alkoxymethyl-4-hydroxyphenyl)propan-1-one (1-15)} (Čižmáriková et al., 1991)

To a sulfonation flask setup with mechanical stirring, reflux condenser and contact thermometer, $0.12 \mathrm{~mol}$ (chloro4-hydroxyphenyl)propan-1-one and $100 \mathrm{~cm}^{3}$ of dried corresponding alcohol were added. The temperature was raised to $40-50^{\circ} \mathrm{C}$, and $19.2 \mathrm{~g}(0.23 \mathrm{~mol})$ of sodium hydrogen<smiles>CCC(=O)c1ccc(O)cc1</smiles>

1a

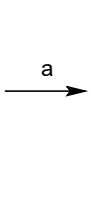

$1 b$<smiles>CCC(=O)c1ccc(O)c(CCl)c1</smiles><smiles>[R]OCc1cc(C(=O)CC)ccc1O</smiles>

$1-15$<smiles>CC(=O)c1ccc(O)cc1</smiles><smiles>CC(=O)c1ccc(O)c(CCl)c1</smiles>

27

28

Figure 1. Synthetic route for derivatives of propiophenone and chemical structures of derivatives of acetophenone.

carbonate was added gradually during $1 \mathrm{~h}$. The products were crystallised from heptane.

(2RS)- bis[3-(4-acetyl-2-propoxymethyl)phenoxy-2hydroxypropyl]isopropylammonium fumarate

$0.57 \mathrm{~mol}$ chloromethyloxirane was gradually added to a solution of $0.55 \mathrm{~mol}$ (3-alkoxymethyl-4-hydroxyphenyl) ethanone in $0.59 \mathrm{~mol}$ potassium hydroxide dissolved in $50 \mathrm{~cm}^{3}$ water. The stirred mixture was left to react at room temperature for $24 \mathrm{~h}$, the product was extracted with diethyl ether or chloroform, the extract was washed with $5 \%$ sodium hydroxide and water. The organic layer was dried with magnesium sulphate, and the solvent was evaporated. The residue formed by 4-(2,3-epoxypropoxy)-3-(alkoxymethyl) ethanone (cca. $60 \%$ yield) was dissolved without previous purification in ethanol or propan-1-ol $\left(50 \mathrm{~cm}^{3}\right)$ and reacted with isopropylamine $\left(10 \mathrm{~cm}^{3}\right)$. The mixture was kept at $30^{\circ} \mathrm{C}$ for $3 \mathrm{~h}$ and then under reflux for $4 \mathrm{~h}$. The solvent and unreacted isopropylamine was removed under reduced pressure, the residue was diluted with water $\left(25 \mathrm{~cm}^{3}\right)$ and the base was extracted to diethyl ether. The extract was dried with potassium carbonate. Addition of an ethereal solution of fumaric acid resulted in separation of the salt, which was crystallised from an appropriate solvent.

\section{EP1 1-(3-methoxymethyl-4-hydroxyphenyl)propan-1-one} (1)

$\mathrm{C}_{11} \mathrm{H}_{14} \mathrm{O}_{3} \mathrm{M}_{\mathrm{r}}$ 194.23, Anal. calcd. \%C 68.02, \% 7.27 found \%C $68.30, \% \mathrm{H}$ 7.20. Yield: $63 \%, \mathrm{R}_{\mathrm{F}} 0.53, \mathrm{Mp} .78-80^{\circ} \mathrm{C}$ (heptane), IR $\left(\mathrm{cm}^{-1}\right) 3,236\left(\mathrm{rOH}_{\mathrm{aso}}\right), 1,653(\mathrm{vC}=\mathrm{O}), 1,596(\mathrm{vC}=\mathrm{C}), 1,274$ ( $\mathrm{rCOC}), \mathrm{UV}\left(\mathrm{CH}_{3} \mathrm{OH}, \lambda\right.$ in $\mathrm{nm}, \varepsilon$ in $\left.\mathrm{m}^{2} \cdot \mathrm{mol}^{-1}\right) \lambda_{\max } 222 \mathrm{~nm}\left(\log \varepsilon_{1}\right.$ 3.24), $273\left(\log \varepsilon_{2} 3.25\right)$

'H-NMR $\left(\mathrm{CDCl}_{3}\right)$ : 1.18-1.23 (t, 3H, $\left.\mathrm{COCH}_{2} \mathrm{CH}_{3}\right), 2.90-2.99$ (q $\left.2 \mathrm{H}, \mathrm{COCH}_{2} \mathrm{CH}_{3}\right), 3.47\left(\mathrm{~s}, 3 \mathrm{H}, \mathrm{CH}_{2} \mathrm{OCH}_{3}\right), 4.72\left(\mathrm{~s}, 2 \mathrm{H}, \mathrm{Ar}-\mathrm{CH}_{2} \mathrm{O}\right)$, 6.89-6.92 (d, 1H, $\left.\mathrm{CH}_{\mathrm{AR}}{ }^{6}\right), 7.70-7.71\left(\mathrm{~d}, 1 \mathrm{H}, \mathrm{CH}_{\mathrm{AR}}{ }^{3}\right), 7.83-7.87$ $\left(\mathrm{dd}, 1 \mathrm{H}, \mathrm{CH}_{\mathrm{AR}}^{5}\right), 8.16(\mathrm{~s}, 1 \mathrm{H}, \mathrm{ArOH})$ 
<smiles>O=C(O)c1ccc(O)c(CO)c1</smiles>

Table 1. Overview of the studied 1-[3-(alkoxymethyl)-4-hydroxyphenyl]alkanones.

\begin{tabular}{|c|c|c|c|}
\hline Substance Number & Description & R1 & $\mathbf{R 2}$ \\
\hline 1 & EP1 & $\mathrm{CH}_{2} \mathrm{CH}_{3}$ & $\mathrm{CH}_{3}$ \\
\hline 2 & EP2 & $\mathrm{CH}_{2} \mathrm{CH}_{3}$ & $\mathrm{CH}_{2} \mathrm{CH}_{3}$ \\
\hline 3 & EP3 & $\mathrm{CH}_{2} \mathrm{CH}_{3}$ & $\left(\mathrm{CH}_{2}\right)_{2} \mathrm{CH}_{3}$ \\
\hline 4 & EP3i & $\mathrm{CH}_{2} \mathrm{CH}_{3}$ & $\mathrm{CH}\left(\mathrm{CH}_{3}\right)_{2}$ \\
\hline 5 & EP4n & $\mathrm{CH}_{2} \mathrm{CH}_{3}$ & $\left(\mathrm{CH}_{2}\right)_{3} \mathrm{CH}_{3}$ \\
\hline 6 & EP4i & $\mathrm{CH}_{2} \mathrm{CH}_{3}$ & $\mathrm{CH}_{2} \mathrm{CH}\left(\mathrm{CH}_{3}\right)_{2}$ \\
\hline 7 & EP5n & $\mathrm{CH}_{2} \mathrm{CH}_{3}$ & $\left(\mathrm{CH}_{2}\right)_{4} \mathrm{CH}_{3}$ \\
\hline 8 & EP5i & $\mathrm{CH}_{2} \mathrm{CH}_{3}$ & $\left(\mathrm{CH}_{2}\right)_{2} \mathrm{CH}\left(\mathrm{CH}_{3}\right)_{2}$ \\
\hline 9 & EP6n & $\mathrm{CH}_{2} \mathrm{CH}_{3}$ & $\left(\mathrm{CH}_{2}\right)_{5} \mathrm{CH}_{3}$ \\
\hline 10 & EP7n & $\mathrm{CH}_{2} \mathrm{CH}_{3}$ & $\left(\mathrm{CH}_{2}\right)_{6} \mathrm{CH}_{3}$ \\
\hline 11 & EP8n & $\mathrm{CH}_{2} \mathrm{CH}_{3}$ & $\left(\mathrm{CH}_{2}\right)_{7} \mathrm{CH}_{3}$ \\
\hline 12 & EP9n & $\mathrm{CH}_{2} \mathrm{CH}_{3}$ & $\left(\mathrm{CH}_{2}\right)_{8} \mathrm{CH}_{3}$ \\
\hline 13 & EP10n & $\mathrm{CH}_{2} \mathrm{CH}_{3}$ & $\left(\mathrm{CH}_{2}\right)_{9} \mathrm{CH}_{3}$ \\
\hline 14 & EP5C & $\mathrm{CH}_{2} \mathrm{CH}_{3}$ & Cyclopentyl \\
\hline 15 & EPbenz & $\mathrm{CH}_{2} \mathrm{CH}_{3}$ & $\mathrm{CH}_{2}$ phenyl \\
\hline 16 & EA1 & $\mathrm{CH}_{3}$ & $\mathrm{CH}_{3}$ \\
\hline 17 & EA2 & $\mathrm{CH}_{3}$ & $\mathrm{CH}_{2} \mathrm{CH}_{3}$ \\
\hline 18 & EA3n & $\mathrm{CH}_{3}$ & $\mathrm{CH}_{2} \mathrm{CH}_{2} \mathrm{CH}_{3}$ \\
\hline 19 & EA3i & $\mathrm{CH}_{3}$ & $\left.\mathrm{CH}_{(} \mathrm{CH}_{3}\right)_{2}$ \\
\hline 20 & EA4n & $\mathrm{CH}_{3}$ & $\left(\mathrm{CH}_{2}\right)_{3} \mathrm{CH}_{3}$ \\
\hline 21 & EA5n & $\mathrm{CH}_{3}$ & $\left(\mathrm{CH}_{2}\right)_{4} \mathrm{CH}_{3}$ \\
\hline 22 & EA7n & $\mathrm{CH}_{3}$ & $\left(\mathrm{CH}_{2}\right)_{6} \mathrm{CH}_{3}$ \\
\hline 23 & EA8n & $\mathrm{CH}_{3}$ & $\left(\mathrm{CH}_{2}\right)_{7} \mathrm{CH}_{3}$ \\
\hline 24 & EA9n & $\mathrm{CH}_{3}$ & $\left(\mathrm{CH}_{2}\right)_{8} \mathrm{CH}_{3}$ \\
\hline 25 & EA5C & $\mathrm{CH}_{3}$ & Cyclopentyl \\
\hline 26 & EAbenz & $\mathrm{CH}_{3}$ & $\mathrm{CH}_{2}$ phenyl \\
\hline 27 & 4-OHacet & & \\
\hline 28 & EAch & & \\
\hline
\end{tabular}

${ }^{13} \mathrm{C}-\mathrm{NMR}\left(\mathrm{CDCl}_{3}\right): 8.45\left(\mathrm{COCH}_{2} \mathrm{CH}_{3}\right), 31.34\left(\mathrm{COCH}_{2} \mathrm{CH}_{3}\right), 58.49$ $\left(\mathrm{CH}_{2} \mathrm{OCH}_{3}\right), 73.97\left(\mathrm{Ar}-\mathrm{CH}_{2} \mathrm{O}\right), 116.39\left(\mathrm{C}_{\mathrm{AR}}{ }^{6}\right), 121.90\left(\mathrm{C}_{\mathrm{AR}}{ }^{3}\right), 128.53$ $\left(C_{A R}{ }^{2}\right), 129.32\left(C_{A R}^{5}\right), 130.19\left(C_{A R}{ }^{4}\right), 160.60\left(C_{A R}{ }^{1}\right), 199.43(C O)$

EP2 1-(3-ethoxymethyl-4-hydroxyphenyl)propan-1-one (2) $\mathrm{C}_{12} \mathrm{H}_{16} \mathrm{O}_{3} \mathrm{M}_{\mathrm{r}}$ 208.26, Anal. calcd. \%C 69.21, \%H 7.74 found \% C69.01, \%H 7.93. Yield: $57 \%, \mathrm{R}_{\mathrm{F}} 0.52, \mathrm{Mp} .65-67^{\circ} \mathrm{C}$ (heptane), IR $\left(\mathrm{cm}^{-1}\right)$ 3,236 $\left(\mathrm{rOH}_{\text {asoc }}\right), 1,653(\mathrm{vC}=\mathrm{O}), 1,596(\mathrm{vC}=\mathrm{C}), 1,274$
(vCOC), UV $\left(\mathrm{CH}_{3} \mathrm{OH}, \lambda\right.$ in nm, $\varepsilon$ in $\left.\mathrm{m}^{2} \cdot \mathrm{mol}^{-1}\right) ; \lambda_{\max } 201 \mathrm{~nm}\left(\log \varepsilon_{1}\right.$ 3.29), $216\left(\log \varepsilon_{2} 3.26\right), 264\left(\log \varepsilon_{3} 3.22\right)$

${ }^{1} \mathrm{H}-\mathrm{NMR}\left(\mathrm{CDCl}_{3}\right): 1.17-1.21\left(\mathrm{t}, 3 \mathrm{H}, \mathrm{COCH}_{2} \mathrm{CH}_{3}\right), 1.22-1.26(\mathrm{t}, 3 \mathrm{H}$, $\left.\mathrm{CH}_{3 \mathrm{alk}}{ }^{2}\right), 2.92-2.95\left(\mathrm{q}, 2 \mathrm{H}, \mathrm{COCH}_{2} \mathrm{CH}_{3}\right), 3.59-3.67\left(\mathrm{q}, 2 \mathrm{H}, \mathrm{CH}_{2 \text { alk }}{ }^{1}\right)$, $4.76\left(\mathrm{~s}, 2 \mathrm{H}, \mathrm{Ar}-\mathrm{CH}_{2} \mathrm{O}\right), 6.89-6.92\left(\mathrm{~d}, 1 \mathrm{H}, \mathrm{CH}_{\mathrm{AR}}{ }^{6}\right), 7.69-7.70(\mathrm{~d}, 1 \mathrm{H}$, $\left.\mathrm{CH}_{\mathrm{AR}}{ }^{3}\right), 7.82-7.85\left(\mathrm{dd}, 1 \mathrm{H}, \mathrm{CH}_{\mathrm{AR}}{ }^{5}\right), 8.39(\mathrm{~s}, 1 \mathrm{H}, \mathrm{ArOH})$

${ }^{13} \mathrm{C}-\mathrm{NMR} \quad\left(\mathrm{CDCl}_{3}\right): 9.08\left(\mathrm{COCH}_{2} \mathrm{CH}_{3}\right), 15.60\left(\mathrm{C}_{\mathrm{alk}}{ }^{2}\right), 32.28$ $\left(\mathrm{COCH}_{2} \mathrm{CH}_{3}\right), 67.25\left(\mathrm{Ar}-\mathrm{CH}_{2} \mathrm{O}\right), 68.60\left(\mathrm{C}_{\mathrm{alk}}{ }^{1}\right), 115.89\left(\mathrm{C}_{\mathrm{AR}}{ }^{6}\right), 126.33$ 
$\left(C_{A R}{ }^{2}\right), 129.89\left(C_{A R}^{5}\right), 130.91\left(C_{A R}^{4}\right), 131.12\left(C_{A R}^{3}\right), 161.53\left(C_{A R}{ }^{1}\right)$, $202.23(C O)$

EP3n 1-(4-hydroxy-3-propoxymethylphenyl)propan-1one (3)

$\mathrm{C}_{13} \mathrm{H}_{18} \mathrm{O}_{3} \mathrm{M}_{\mathrm{R}}=222.29$ Anal. calcd. \%C $70.24 \% \mathrm{H} 8.16$ found \%C 70.54, \%H 8.32. Yield: $65 \%, \mathrm{R}_{\mathrm{F}} 0.55$ (Mp. $61-63^{\circ} \mathrm{C}$ (heptane), IR $\left(\mathrm{cm}^{-1}\right), 3,248\left(\mathrm{rOH}_{\text {asoc }}\right), 1,686(\mathrm{vC}=\mathrm{O}), 1,602(\mathrm{vC}=\mathrm{C}), 1,272$ (vCOC), UV $\left(\mathrm{CH}_{3} \mathrm{OH}, \lambda\right.$ in $\mathrm{nm} \varepsilon$ in $\left.\mathrm{m}^{2} \cdot \mathrm{mol}^{-1}\right) ; \lambda_{\max } 227 \mathrm{~nm}\left(\log \varepsilon_{1}\right.$ 3.01), 264 ( $\left.\log \varepsilon_{2} 3.132\right), 277\left(\log \varepsilon_{3} 3.10\right)$

${ }^{1} \mathrm{H}-\mathrm{NMR}\left(\mathrm{CDCl}_{3}\right): 0.93-0.98\left(\mathrm{t}, 3 \mathrm{H}, \mathrm{CH}_{3 \text { alk }}{ }^{3}\right), 1.13-1.18(\mathrm{t}, 3 \mathrm{H}$, $\left.\mathrm{COCH}_{2} \mathrm{CH}_{3}\right), 1.61-1.68\left(\mathrm{~m}, 2 \mathrm{H}, \mathrm{CH}_{3 \text { alk }}{ }^{2}\right), 2.92-3.00(\mathrm{q}, 2 \mathrm{H}$, $\left.\left.\mathrm{COCH}_{2} \mathrm{CH}_{3}\right), 3.51-3.55\left(\mathrm{t}, 2 \mathrm{H}, \mathrm{CH}_{3 \text { alk }}\right)^{1}\right), 4.75\left(\mathrm{~s}, 2 \mathrm{H}, \mathrm{Ar}-\mathrm{CH}_{2} \mathrm{O}\right)$, 6.82-6.85 (d, 1H, $\left.\mathrm{CH}_{\mathrm{AR}}{ }^{6}\right), 7.69-7.70\left(\mathrm{~d}, 1 \mathrm{H}, \mathrm{CH}_{\mathrm{AR}}{ }^{3}\right), 7.82-7.91$ (dd, $\left.1 \mathrm{H}, \mathrm{CH}_{\mathrm{AR}}{ }^{5}\right), 8.43(\mathrm{~s}, 1 \mathrm{H}, \mathrm{ArOH})$

${ }^{13} \mathrm{C}-\mathrm{NMR}\left(\mathrm{CDCl}_{3}\right)$ : $9.08\left(\mathrm{COCH}_{2} \mathrm{CH}_{3}\right), 11.12\left(\mathrm{C}_{\mathrm{alk}}{ }^{3}\right), 24.05\left(\mathrm{C}_{\mathrm{alk}}{ }^{2}\right)$, $32.28\left(\mathrm{COCH}_{2} \mathrm{CH}_{3}\right), 68.74\left(\mathrm{Ar}-\mathrm{CH}_{2} \mathrm{O}\right), 73.62\left(\mathrm{C}_{\mathrm{alk}}{ }^{1}\right), 115.87\left(\mathrm{C}_{\mathrm{AR}}{ }^{6}\right)$, $126.41\left(C_{A R}{ }^{2}\right), 129.86\left(C_{A R}^{5}\right), 130.85\left(C_{A R}^{4}\right), 131.02\left(C_{A R}{ }^{3}\right), 161.74$ $\left(C_{A R}{ }^{1}\right), 199.81(C O)$

EP3i 1-(4-hydroxy-3-isopropoxymethylphenyl)propan-1one (4)

$\mathrm{C}_{13} \mathrm{H}_{18} \mathrm{O}_{3} \mathrm{M}_{\mathrm{r}} 222.29$ Anal. calcd. \% C 70.24\% H 8.16 found \% C $70.54, \% \mathrm{H}$ 8.32. Yield: $65 \%, \mathrm{R}_{\mathrm{F}} 0.55, \mathrm{Mp} .61-63^{\circ} \mathrm{C}$ (heptane), IR $\left(\mathrm{cm}^{-1}\right), 3,248\left(\mathrm{rOH}_{\text {asoc. }}\right), 1,686(\mathrm{rC}=\mathrm{O}), 1,602(\mathrm{rC}=\mathrm{C}), 1,272$ (vCOC), UV $\left(\mathrm{CH}_{3} \mathrm{OH}, \lambda\right.$ in $\mathrm{nm}, \varepsilon$ in $\left.\mathrm{m}^{2} \cdot \mathrm{mol}^{-1}\right) ; \lambda_{\max } 226 \mathrm{~nm}\left(\log \varepsilon_{1}\right.$ 3.14), $263\left(\log \varepsilon_{2} 3.16\right), 276\left(\log \varepsilon_{3} 3.12\right)$

'H-NMR: $1.17-1.23\left(\mathrm{t}, 3 \mathrm{H}, \mathrm{COCH}_{2} \mathrm{CH}_{3}\right), 1.25-1.27(\mathrm{~d}, 6 \mathrm{H}$, $\left.\mathrm{CH}\left(\mathrm{CH}_{3}\right)_{2}\right), 2.89-2.97\left(\mathrm{q}, 2 \mathrm{H}, \mathrm{COCH}_{2} \mathrm{CH}_{3}\right), 3.74-3.82(\mathrm{~m}, 1 \mathrm{H}, \mathrm{CH})$, $4.76\left(\mathrm{~s}, 2 \mathrm{H}, \mathrm{Ar}-\mathrm{CH}_{2} \mathrm{O}\right), 6.88-6.91\left(\mathrm{~d}, 1 \mathrm{H}, \mathrm{CH}_{\mathrm{AR}}{ }^{6}\right), 7.68-7.69(\mathrm{~d}, 1 \mathrm{H}$, $\left.\mathrm{CH}_{\mathrm{AR}}{ }^{3}\right), 7.81-7.84\left(\mathrm{dd}, 1 \mathrm{H}, \mathrm{CH}_{\mathrm{AR}}{ }^{5}\right), 8.57(\mathrm{~s}, 1 \mathrm{H}, \mathrm{ArOH})$

${ }^{13} \mathrm{C}-\mathrm{NMR}: \quad 9.07 \quad(\mathrm{COCH} 2 \mathrm{CH} 3), \quad 22.55 \quad\left(\mathrm{CH}\left(\mathrm{CH}_{3}\right)_{2}\right), \quad 32.23$ $\left(\mathrm{COCH}_{2} \mathrm{CH}_{3}\right), 66.25\left(\mathrm{Ar}-\mathrm{CH}_{2} \mathrm{O}\right), 73.04(\mathrm{CH}), 115.80\left(\mathrm{C}_{\mathrm{AR}}{ }^{6}\right), 126.70$ $\left(C_{A R}{ }^{3}\right), 129.82\left(C_{A R}{ }^{4}\right), 130,72\left(C_{A R}^{5}\right), 130.90\left(C_{A R}{ }^{2}\right), 161.39\left(C_{A R}{ }^{1}\right)$, $202.11(C O)$

EP4n 1-(3-butyloxymethyl-4-hydroxyphenyl)propan-1one (5)

$\mathrm{C}_{14} \mathrm{H}_{20} \mathrm{O}_{3} \mathrm{M}_{\mathrm{r}}=236.36$, Anal. calcd. \%C $71.16 \% \mathrm{H} 8.53$ found \%C 71.40, \%H 8.52. Yield: $69 \%, \mathrm{R}_{\mathrm{F}} 0.57, \mathrm{Mp} .68-70^{\circ} \mathrm{C}$ (heptane), IR $\left(\mathrm{cm}^{-1}\right), 3,340\left(\mathrm{rOH}_{\text {asoc }}\right), 1,684(\mathrm{rC}=\mathrm{O}), 1,600(\mathrm{rC}=\mathrm{C}), 1,270$ (vCOC), UV $\left(\mathrm{CH}_{3} \mathrm{OH}, \lambda\right.$ in $\mathrm{nm}, \varepsilon$ in $\left.\mathrm{m}^{2} \cdot \mathrm{mol}^{-1}\right) ; \lambda_{\max } 201$ (log $\varepsilon_{1}$ 3.23), $217\left(\log \varepsilon_{2} 3.22\right), 264\left(\log \varepsilon_{3} 3.13\right) ; 277\left(\log \varepsilon_{4} 3.20\right)$

${ }^{1} \mathrm{H}-\mathrm{NMR}\left(\mathrm{CDCl}_{3}\right): 0.91-0.96\left(\mathrm{t}, 3 \mathrm{H}, \mathrm{CH}_{3}{ }^{4}{ }^{4}\right), 1.13-1.18(\mathrm{t}, 3 \mathrm{H}$, $\left.\mathrm{COCH}_{2} \mathrm{CH}_{3}\right), 1.41-1.46\left(\mathrm{~m}, 2 \mathrm{H}, \mathrm{CH}_{2 \text { alk }}{ }^{3}\right), 1.56-1.64\left(\mathrm{~m}, 2 \mathrm{H}, \mathrm{CH}_{2}\right.$ $\left.{ }_{\text {alk }}^{2}\right)$, 2.92-3.00 (m, 2H, $\left.\mathrm{COCH}_{2} \mathrm{CH}_{3}\right), 3.52-3.57\left(\mathrm{t}, 2 \mathrm{H}, \mathrm{CH}_{\text {2alk }}{ }^{1}\right)$, $4.54\left(\mathrm{~s}, 2 \mathrm{H}, \mathrm{Ar}-\mathrm{CH}_{2} \mathrm{O}\right), 6.81-6.86\left(\mathrm{~d}, 1 \mathrm{H}, \mathrm{CH}_{\mathrm{AR}}{ }^{6}\right), 7.80-7.86(\mathrm{~d}, 1 \mathrm{H}$, $\left.\mathrm{CH}_{\mathrm{AR}}{ }^{3}\right), 7.95-7.98\left(\mathrm{dd}, 1 \mathrm{H}, \mathrm{CH}_{\mathrm{AR}}^{5}\right), 8.39(\mathrm{~s}, 1 \mathrm{H}, \mathrm{ArOH})$

${ }^{13} \mathrm{C}-\mathrm{NMR}\left(\mathrm{CDCl}_{3}\right): 9.10\left(\mathrm{COCH}_{2} \mathrm{CH}_{3}\right), 14.39\left(\mathrm{C}^{\mathrm{alk} 4}\right), 20.55\left(\mathrm{C}_{\mathrm{alk}}{ }^{3}\right)$, $32.25\left(\mathrm{C}_{\mathrm{alk}}{ }^{2}\right), 33.02\left(\mathrm{COCH}_{2} \mathrm{CH}_{3}\right), 68.78\left(\mathrm{Ar}-\mathrm{CH}_{2} \mathrm{O}\right), 71.67\left(\mathrm{C}_{\mathrm{alk}}{ }^{1}\right)$, $115.88\left(C_{A R}{ }^{6}\right), 126.41\left(C_{A R}{ }^{2}\right), 129.87\left(C_{A R}^{5}\right), 130.85\left(C_{A R}{ }^{4}\right), 131.04$ $\left(C_{A R}{ }^{3}\right), 161.51\left(C_{A R}{ }^{1}\right), 202.21(C O)$
EP4i 1-(4-hydroxy-3-isobutyloxymethylphenyl)propan-1one (6)

$\mathrm{C}_{14} \mathrm{H}_{20} \mathrm{O}_{3} \mathrm{M}_{\mathrm{r}} 236.36$ Anal. calcd. \%C $71.97 \% \mathrm{H} 8.86$ found \%C 72.30, \%H 8.70. Yield: $62 \%, \mathrm{R}_{\mathrm{F}} 0.62, \mathrm{Mp} .52-54^{\circ} \mathrm{C}$ (heptane), IR $\left(\mathrm{cm}^{-1}\right) 3344\left(\mathrm{vOH}_{\text {asoc }}\right), 1656(\mathrm{vC}=\mathrm{O}), 1592(\mathrm{vC}=\mathrm{C}), 1277$ (vCOC), UV $\left(\mathrm{CH}_{3} \mathrm{OH}, \lambda\right.$ in $\mathrm{nm}, \varepsilon$ in $\left.\mathrm{m}^{2} \cdot \mathrm{mol}^{-1}\right) ; \lambda_{\max } 203$ (log $\left.\varepsilon_{1} 3.26\right), 222$ ( $\left.\log \varepsilon_{2} 3.28\right), 274\left(\log \varepsilon_{3} 3.20\right)$

${ }^{1} \mathrm{H}-\mathrm{NMR}\left(\mathrm{CDCl}_{3}\right): 0.94-0.96\left(\mathrm{~d}, 6 \mathrm{H}, \mathrm{CH}\left(\mathrm{CH}_{3}\right)_{2}\right), 1.19-1.21(\mathrm{t}, 3 \mathrm{H}$, $\left.\mathrm{COCH}_{2} \mathrm{CH}_{3}\right), 1.90-1.99\left(\mathrm{~m}, 1 \mathrm{H}, \mathrm{CH}\left(\mathrm{CH}_{3}\right)_{2}\right), 2.92-2.67(\mathrm{q}, 2 \mathrm{H}$, $\left.\mathrm{COCH}_{2} \mathrm{CH}_{3}\right), 4.75\left(\mathrm{~s}, 2 \mathrm{H}, \mathrm{Ar}-\mathrm{CH}_{2} \mathrm{O}\right), 6.89-6.90\left(\mathrm{~d}, 1 \mathrm{H}, \mathrm{CH}_{\mathrm{AR}}{ }^{6}\right)$, 7.68-7.69 (d, $\left.1 \mathrm{H}, \mathrm{CH}_{\mathrm{AR}}{ }^{3}\right), 7.82-7.92\left(\mathrm{dd}, 1 \mathrm{H}, \mathrm{CH}_{\mathrm{AR}}{ }^{3}\right), 8.38(\mathrm{~s}, 1 \mathrm{H}$, $\mathrm{ArOH})$

${ }^{13} \mathrm{C}-\mathrm{NMR}\left(\mathrm{CDCl}_{3}\right): 9.11\left(\mathrm{COCH}_{2} \mathrm{CH}_{3}\right), 19.91\left(\mathrm{CH}\left(\mathrm{CH}_{3}\right)_{2}\right), 29.80$ $\left(\mathrm{CH}\left(\mathrm{CH}_{3}\right)_{2}\right), 32.29\left(\mathrm{COCH}_{2} \mathrm{CH}_{3}\right), 68.91\left(\mathrm{Ar}-\mathrm{CH}_{2} \mathrm{O}\right), 78.84\left(\mathrm{CH}_{2}-\right.$ $\mathrm{CH}), 115.86\left(\mathrm{C}_{\mathrm{AR}}{ }^{6}\right), 126.52\left(\mathrm{C}_{\mathrm{AR}}{ }^{2}\right), 129.87\left(\mathrm{C}_{\mathrm{AR}}{ }^{5}\right), 130.79\left(\mathrm{C}_{\mathrm{AR}}{ }^{4}\right)$, $130.92\left(C_{A R}{ }^{3}\right), 161.47\left(C_{A R}{ }^{1}\right), 202.25(C O)$

EP5n 1-(4-hydroxy-3-pentyloxymethylphenyl)propan-1one (7)

$\mathrm{C}_{15} \mathrm{H}_{22} \mathrm{O}_{3} \mathrm{M}_{\mathrm{r}} 250.34$, Anal. calcd. \% C $71.97 \% \mathrm{H} 8.86$ found \%C 72.20, \%H 8.60. Yield: 68\%, R $0.60, \mathrm{Mp} .30-32^{\circ} \mathrm{C}$ (heptane), IR $\left(\mathrm{cm}^{-1}\right) 3340\left(\mathrm{vOH}_{\text {asoc }}\right), 1684(\mathrm{vC}=\mathrm{O}), 1604(\mathrm{vC}=\mathrm{C}), 1272$ (vCOC). UV $\left(\mathrm{CH}_{3} \mathrm{OH}, \lambda\right.$ in nm, $\varepsilon$ in $\left.\mathrm{m}^{2} \cdot \mathrm{mol}^{-1}\right) ; \lambda_{\max } 202$ (log $\left.\varepsilon_{1} 2.90\right), 222$ $\mathrm{nm}\left(\log \varepsilon_{2} 3.25\right), 274\left(\log \varepsilon_{3} 3.21\right)$

${ }^{1} \mathrm{H}-\mathrm{NMR}\left(\mathrm{CDCl}_{3}\right)$ : 0.88-0.93 (t, 3H, $\left.\mathrm{CH}_{3 \text { alk }}{ }^{5}\right), 1.18-1.23(\mathrm{t}, 3 \mathrm{H}$, $\left.\mathrm{COCH}_{2} \mathrm{CH}_{3}\right), 1.32-1.35\left(\mathrm{~m}, 4 \mathrm{H}, \mathrm{CH}_{2 \text { alk }}{ }^{3,4}\right), 1.61-1.70\left(\mathrm{~m}, 2 \mathrm{H}, \mathrm{CH}_{2}\right.$ alk $\left.{ }^{2}\right), 2.90-2.97\left(\mathrm{q}, 2 \mathrm{H}, \mathrm{COCH}_{2} \mathrm{CH}_{3}\right), 3.54-3.58\left(\mathrm{t}, 2 \mathrm{H}, \mathrm{CH}_{2}{ }_{\text {alk }}{ }^{2}\right)$, $4.75\left(\mathrm{~s}, 2 \mathrm{H}, \mathrm{Ar}-\mathrm{CH}_{2} \mathrm{O}\right), 6.89-6.92\left(\mathrm{~d}, 1 \mathrm{H}, \mathrm{CH}_{\mathrm{AR}}{ }^{6}\right), 7.69-7.70(\mathrm{~d}, 1 \mathrm{H}$, $\left.\mathrm{CH}_{\mathrm{AR}}{ }^{3}\right), 7.82-7.90\left(\mathrm{dd}, 1 \mathrm{H}, \mathrm{CH}_{\mathrm{AR}}{ }^{5}\right), 8.41(\mathrm{~s}, 1 \mathrm{H}, \mathrm{ArOH})$

${ }^{13} \mathrm{C}-\mathrm{NMR}\left(\mathrm{CDCl}_{3}\right): 9.08\left(\mathrm{COCH}_{2} \mathrm{CH}_{3}\right), 14.57\left(\mathrm{C}_{\mathrm{al}} \mathrm{k}^{5}\right), 23.70\left(\mathrm{C}_{\mathrm{alk}}{ }^{4}\right)$, $29.65\left(\mathrm{C}_{\mathrm{alk}}{ }^{3}\right), 30.57\left(\mathrm{C}_{\mathrm{alk}}{ }^{2}\right), 32.26\left(\mathrm{COCH}_{2} \mathrm{CH}_{3}\right), 68.76\left(\mathrm{Ar}-\mathrm{CH}_{2} \mathrm{O}\right)$, 71.95 (Calk'), $115.85\left(C_{A R}{ }^{6}\right), 126.37\left(C_{A R}{ }^{2}\right), 129.82\left(C_{A R}{ }^{5}\right), 130.80$ $\left(C_{A R}{ }^{4}\right), 130.98\left(C_{A R}{ }^{3}\right), 161.46\left(C_{A R}{ }^{1}\right), 202.11(C O)$

\section{EP5i 1-(4-hydroxy-3-isopentyloxymethylphenyl)propan- 1-one (8)}

$\mathrm{C}_{15} \mathrm{H}_{22} \mathrm{O}_{3} \mathrm{M}_{\mathrm{r}} 250.34$, Anal. calcd. \% C $71.97 \% \mathrm{H} 8.86$ found \%C 72.30, \% $\mathrm{H}$ 8.70. Yield: $63 \%, \mathrm{R}_{\mathrm{F}} 0.58, \mathrm{Mp} .68-70^{\circ} \mathrm{C}$ (heptane), IR $\left(\mathrm{cm}^{-1}\right), 3,342\left(\mathrm{vOH}_{\text {asoc }}\right), 1,683(\mathrm{vC}=\mathrm{O}), 1,604(\mathrm{vC}=\mathrm{C}), 1,273$ ( $\mathrm{vCOC}), \mathrm{UV}\left(\mathrm{CH}_{3} \mathrm{OH}, \lambda\right.$ in nm, $\varepsilon$ in $\left.\mathrm{m}^{2} \cdot \mathrm{mol}^{-1}\right) ; \lambda_{\max } 204$ (log $\varepsilon_{1}$ 3.18), $223 \mathrm{~nm}\left(\log \varepsilon_{2} 3.15\right), 274\left(\log \varepsilon_{3} 3.23\right)$

${ }^{1} \mathrm{H}-\mathrm{NMR}\left(\mathrm{CDCl}_{3}\right)$ : 0.88-0.90 (d, 6H, CH( $\left.\left(\mathrm{CH}_{3}\right)_{2}\right), 1.18-1.20(\mathrm{t}, 3 \mathrm{H}$, $\left.\mathrm{COCH}_{2} \mathrm{CH}_{3}\right), 1.51-1.56\left(\mathrm{~m}, 1 \mathrm{H}, \mathrm{CH}\left(\mathrm{CH}_{3}\right)_{2}\right), 2.92-2.95(\mathrm{q}, 2 \mathrm{H}$, $\left.\mathrm{COCH}_{2} \mathrm{CH}_{3}\right), 4.75\left(\mathrm{~s}, 2 \mathrm{H}, \mathrm{Ar}-\mathrm{CH}_{2} \mathrm{O}\right), 6.89-6.92\left(\mathrm{~d}, 1 \mathrm{H}, \mathrm{CH}_{\mathrm{AR}}{ }^{6}\right)$, 7.69-7.70 (d, 1H, $\left.\mathrm{CH}_{A R}{ }^{3}\right), 7.82-7.86\left(\mathrm{dd}, 1 \mathrm{H}, \mathrm{CH}_{\mathrm{AR}}{ }^{5}\right), 8.40(\mathrm{~s}, 1 \mathrm{H}$, $\mathrm{ArOH})$

${ }^{13} \mathrm{C}-\mathrm{NMR}\left(\mathrm{CDCl}_{3}\right): 9.27\left(\mathrm{COCH}_{2} \mathrm{CH}_{3}\right), 19.91\left(\mathrm{CH}\left(\mathrm{CH}_{3}\right)_{2}\right), 29.80$ $\left(\mathrm{CH}\left(\mathrm{CH}_{3}\right)_{2}\right), 32.13\left(\mathrm{COCH}_{2} \mathrm{CH}_{3}\right), 70.49\left(\mathrm{Ar}-\mathrm{CH}_{2} \mathrm{O}\right), 77.82\left(\mathrm{CH}_{2}-\right.$ $\mathrm{CH}), 117.15\left(\mathrm{C}_{\mathrm{AR}}{ }^{6}\right), 123.03\left(\mathrm{C}_{\mathrm{AR}}{ }^{2}\right), 129.11\left(\mathrm{C}_{\mathrm{AR}}{ }^{5}\right), 129.97\left(\mathrm{C}_{\mathrm{AR}}{ }^{4}\right)$, $130.86\left(C_{A R}^{3}\right), 161.50\left(C_{A R}^{1}\right), 200.35(C O)$ 
EP6n 1-(3-hexyloxymethyl-4-hydroxyphenyl)propan-1one (9)

$\mathrm{C}_{16} \mathrm{H}_{24} \mathrm{O}_{3} \mathrm{M}_{\mathrm{r}}$ 264.36, Anal. calcd. \%C $72.69 \% \mathrm{H} 9.15$ found \%C 72.77, \%H 8.90. Yield: $60 \%, \mathrm{R}_{\mathrm{F}} 0.61, \mathrm{Mp} .51-53^{\circ} \mathrm{C}$ (heptane), IR $\left(\mathrm{cm}^{-1}\right), 3,340\left(\mathrm{vOH}_{\text {asoc }}\right), 1,684(\mathrm{rC}=\mathrm{O}), 1,600(\mathrm{vC}=\mathrm{C}), 1,272$ (vCOC). UV $\left(\mathrm{CH}_{3} \mathrm{OH}, \lambda\right.$ in nm, $\varepsilon$ in $\left.\mathrm{m}^{2} \cdot \mathrm{mol}^{-1}\right) \lambda_{\max } 202$ (3.26), 216 $\mathrm{nm}\left(\log \varepsilon_{1} 3.25\right), 264\left(\log \varepsilon_{2} 3.24\right)$

${ }^{1} \mathrm{H}-\mathrm{NMR}\left(\mathrm{CDCl}_{3}\right)$ : 0.89-0.93 (m, 3H, $\left.\mathrm{CH}_{3 \text { alk }}{ }^{6}\right), 1.13-1.17(\mathrm{t}, 3 \mathrm{H}$, $\left.\mathrm{COCH}_{2} \mathrm{CH}_{3}\right), 1.28-1.40\left(\mathrm{~m}, 8 \mathrm{H}, \mathrm{CH}_{2 \text { alk }}^{3,4,5}\right), 1,58-1,62(\mathrm{~m}, 2 \mathrm{H}$, $\left.\mathrm{CH}_{2 \text { alk }}{ }^{2}\right), 2.94-3.01\left(\mathrm{q}, 2 \mathrm{H}, \mathrm{COCH}_{2} \mathrm{CH}_{3}\right), 3.50-3.53\left(\mathrm{t}, 2 \mathrm{H}, \mathrm{CH}_{2 \text { alk }}{ }^{1}\right)$, $4.73\left(\mathrm{~s}, 2 \mathrm{H}, \mathrm{Ar}-\mathrm{CH}_{2} \mathrm{O}\right), 6.82-6.84\left(\mathrm{~d}, 1 \mathrm{H}, \mathrm{CH}_{\mathrm{AR}}{ }^{6}\right), 7.78-7.81(\mathrm{~d}, 1 \mathrm{H}$, $\left.\mathrm{CH}_{\mathrm{AR}}{ }^{3}\right), 7.85-7.88\left(\mathrm{dd}, 1 \mathrm{H}, \mathrm{CH}_{\mathrm{AR}}{ }^{5}\right), 8.39(\mathrm{~s}, 1 \mathrm{H}, \mathrm{ArOH})$

${ }^{13} \mathrm{C}-\mathrm{NMR}\left(\mathrm{CDCl}_{3}\right): 9.13\left(\mathrm{COCH}_{2} \mathrm{CH}_{3}\right), 14.56\left(\mathrm{C}_{\mathrm{alk}}{ }^{6}\right), 23.82\left(\mathrm{C}_{\mathrm{alk}}{ }^{5}\right)$, $27.40\left(\mathrm{C}_{\mathrm{alk}}{ }^{4}\right), 30.40\left(\mathrm{Calk}^{3}\right), 30.90\left(\mathrm{C}_{\mathrm{alk}}{ }^{5}\right), 32.32\left(\mathrm{C}_{\mathrm{alk}}{ }^{2}\right), 33.15$ $\left(\mathrm{COCH}_{2} \mathrm{CH}_{3}\right), 68.75\left(\mathrm{Ar}-\mathrm{CH}_{2} \mathrm{O}\right), 71.93\left(\mathrm{C}_{\mathrm{alk}}{ }^{1}\right), 115.90\left(\mathrm{C}_{\mathrm{AR}}{ }^{6}\right), 126.42$ $\left(C_{A R}{ }^{2}\right), 129.83\left(C_{A R}^{5}\right), 130.85\left(C_{A R}{ }^{4}\right), 131.08\left(C_{A R}{ }^{3}\right), 161.60\left(C_{A R}{ }^{1}\right)$, $202.20(C O)$

EP7n 1-(3-heptyloxymetyl-4-hydroxyphenyl)propan-1one (10)

$\mathrm{C}_{17} \mathrm{H}_{26} \mathrm{O}_{3} \mathrm{M}_{\mathrm{r}} 278.19$, Anal. calcd. \%C $73.35 \% \mathrm{H} 9.41$ found \%C 73.10, \%H 9.20. Yield: $57 \%, \mathrm{R}_{\mathrm{F}} 0.56, \mathrm{Mp} .47-49^{\circ} \mathrm{C}$ (heptane), IR $\left(\mathrm{cm}^{-1}\right), 3,352\left(\mathrm{rOH}_{\text {asoc }}\right), 1,686(\mathrm{rC}=\mathrm{O}), 1,602(\mathrm{vC}=\mathrm{C}), 1,272$ (vCOC), UV ( $\mathrm{CH}_{3} \mathrm{OH}, \lambda$ in nm, $\varepsilon$ in $\left.\mathrm{m}^{2} \cdot \mathrm{mol}^{-1}\right) ; \lambda_{\max } 202$ (3.30), 222 $\mathrm{nm}\left(\log \varepsilon_{1} 3.31\right), 264\left(\log \varepsilon_{2} 3.24\right)$

${ }^{1} \mathrm{H}-\mathrm{NMR}\left(\mathrm{CDCl}_{3}\right)$ : 0.86-0.90 (m, 3H, $\mathrm{CH}_{3}$ alk $\left.^{7}\right), 1.14-1.19(\mathrm{t}, 3 \mathrm{H}$, $\left.\mathrm{COCH}_{2} \mathrm{CH}_{3}\right), 1.29-1.41\left(\mathrm{~m}, 8 \mathrm{H}, \mathrm{CH}_{2}{ }_{\text {alk }}^{3,4,5,6}\right), 1.59-1.64(\mathrm{~m}, 2 \mathrm{H}$, $\left.\mathrm{CH}_{2 \mathrm{alk}}{ }^{2}\right), 2.90-2.97\left(\mathrm{q}, 2 \mathrm{H}, \mathrm{COCH}_{2} \mathrm{CH}_{3}\right), 3.54-3.58\left(\mathrm{t}, 2 \mathrm{H}, \mathrm{CH}_{2 \text { alk }}{ }^{1}\right)$, $4.76\left(\mathrm{~s}, 2 \mathrm{H}, \mathrm{Ar}-\mathrm{CH}_{2} \mathrm{O}\right), 6.89-6.93\left(\mathrm{~d}, 1 \mathrm{H}, \mathrm{CH}_{\mathrm{AR}}{ }^{6}\right), 7.69-7.70(\mathrm{~d}, 1 \mathrm{H}$, $\left.\mathrm{CH}_{\mathrm{AR}}{ }^{5}\right), 7.83-7.86\left(\mathrm{dd}, 1 \mathrm{H}, \mathrm{CH}_{\mathrm{AR}}{ }^{5}\right), 8.38(\mathrm{~s}, 1 \mathrm{H}, \mathrm{ArOH})$

${ }^{13} \mathrm{C}-\mathrm{NMR}\left(\mathrm{CDCl}_{3}\right): 9.11\left(\mathrm{COCH}_{2} \mathrm{CH}_{3}\right), 14.59\left(\mathrm{C}_{\mathrm{alk}}\right), 23.84\left(\mathrm{C}_{\mathrm{alk}}{ }^{6}\right)$, $27.42\left(\mathrm{C}_{\mathrm{alk}}{ }^{4}\right), 30.41\left(\mathrm{Calk}^{3}\right), 30.91\left(\mathrm{C}_{\mathrm{alk}}{ }^{5}\right), 32.30\left(\mathrm{C}_{\mathrm{alk}}{ }^{2}\right), 33.17$ $\left(\mathrm{COCH}_{2} \mathrm{CH}_{3}\right), 68.75\left(\mathrm{Ar}^{-} \mathrm{CH}_{2} \mathrm{O}\right), 71.93\left(\mathrm{C}_{\mathrm{alk}}{ }^{1}\right), 115.92\left(\mathrm{C}_{\mathrm{AR}}{ }^{6}\right), 126.44$ $\left(C_{A R}{ }^{2}\right), 129.85\left(C_{A R}^{5}\right), 130.87\left(C_{A R}^{4}\right), 131.09\left(C_{A R}^{3}\right), 161.61\left(C_{A R}{ }^{1}\right)$, $202.23(\mathrm{CO})$

\section{EP8n 1-(4-hydroxy-3-octyloxymetylphenyl)propan-1-one} (11)

$\mathrm{C}_{18} \mathrm{H}_{28} \mathrm{O}_{3} \mathrm{M}_{\mathrm{r}} 292.45$, Anal. calcd. \%C $73.93 \% \mathrm{H} 9.65$ found \%C $73.70, \% H$ 9.45. Yield: $56 \%, R_{F} 0.57, M p .54-57^{\circ} \mathrm{C}$ (heptane), IR $\left(\mathrm{cm}^{-1}\right), 3,352\left(\mathrm{rOH}_{\text {asoc. }}\right), 1,686(\mathrm{vC}=\mathrm{O}), 1,600(\mathrm{vC}=\mathrm{C}), 1,272$ (vCOC). UV ( $\mathrm{CH}_{3} \mathrm{OH} \lambda$ in nm, $\varepsilon$ in $\left.\mathrm{m}^{2} \cdot \mathrm{mol}^{-1}\right) ; \lambda_{\max } 206$ (3.26), 222 $\mathrm{nm}\left(\log \varepsilon_{1} 3.29\right), 274\left(\log \varepsilon_{2} 3.25\right)$

${ }^{1} \mathrm{H}-\mathrm{NMR}\left(\mathrm{CDCl}_{3}\right)$ : 0.86-0.90 (t, 3H, $\left.\mathrm{CH}_{3 \text { alk }}^{8}\right), 1.18-1.206(\mathrm{t}, 3 \mathrm{H}$, $\left.\mathrm{COCH}_{2} \mathrm{CH}_{3}\right), 1.21-1.28\left(\mathrm{~m}, 10 \mathrm{H}, \mathrm{CH}_{2 \text { alk }}^{3-7}\right), 1.60-1.65(\mathrm{~m}, 2 \mathrm{H}$, $\left.\mathrm{CH}_{\text {2alk }}{ }^{2}\right), 2.90-2.98\left(\mathrm{~m}, 2 \mathrm{H}, \mathrm{COCH}_{2} \mathrm{CH}_{3}\right), 3.54-3.58\left(\mathrm{t}, 2 \mathrm{H}, \mathrm{CH}_{\text {2alk }}{ }^{1}\right)$, $4.75\left(\mathrm{~s}, 2 \mathrm{H}, \mathrm{Ar}-\mathrm{CH}_{2} \mathrm{O}\right), 6.89-6.92\left(\mathrm{~d}, 1 \mathrm{H}, \mathrm{CH}_{\mathrm{AR}}{ }^{6}\right), 7.69-7.92(\mathrm{~d}, 1 \mathrm{H}$, $\left.\mathrm{CH}_{\mathrm{AR}}{ }^{3}\right), 7.83-7.91\left(\mathrm{dd}, 1 \mathrm{H}, \mathrm{CH}_{\mathrm{AR}}{ }^{5}\right), 8.40(\mathrm{~s}, 1 \mathrm{H}, \mathrm{ArOH})$

${ }^{13} \mathrm{C}-\mathrm{NMR}\left(\mathrm{CDCl}_{3}\right): 9.09\left(\mathrm{COCH}_{2} \mathrm{CH}_{3}\right), 14.57\left(\mathrm{C}^{\mathrm{alk} 8}\right), 23.86\left(\mathrm{C}_{\mathrm{alk}}{ }^{7}\right)$, $27.45\left(\mathrm{C}_{\mathrm{alk}}{ }^{6}\right), 30.57\left(\mathrm{C}_{\mathrm{alk}}{ }^{5}\right), 30.67\left(\mathrm{C}_{\mathrm{alk}}{ }^{4}\right), 30.88\left(\mathrm{C}_{\mathrm{alk}}{ }^{3}\right), 32.29\left(\mathrm{C}_{\mathrm{alk}}{ }^{2}\right)$ $33.14\left(\mathrm{COCH}_{2} \mathrm{CH}_{3}\right), 68.73\left(\mathrm{Ar}-\mathrm{CH}_{2} \mathrm{O}\right), 71.91\left(\mathrm{C}_{\mathrm{alk}}{ }^{1}\right), 115.88\left(\mathrm{C}_{\mathrm{AR}}{ }^{6}\right)$, $126.40\left(C_{A R}{ }^{2}\right), 129.86\left(C_{A R}{ }^{5}\right), 130.82\left(C_{A R}{ }^{4}\right), 131.07\left(C_{A R}{ }^{3}\right), 161.51$ $\left(C_{A R}{ }^{1}\right), 202.17(C O)$
EP9n 1-(4-hydroxy-3-nonyloxymethylphenyl)propan-1one (12)

$\mathrm{C}_{19} \mathrm{H}_{30} \mathrm{O}_{3} \mathrm{M}_{\mathrm{r}} 306.45$, Anal. calcd. \%C $74.47 \% \mathrm{H} 9.87$ found \%C $74.10 \% \mathrm{H}$ 9.50. Yield: $67 \%, \mathrm{R}_{\mathrm{F}} 0.60, \mathrm{Mp} .59-61^{\circ} \mathrm{C}$ (heptane), IR $\left(\mathrm{cm}^{-1}\right), 3,343\left(\mathrm{rOH}_{\text {asoc }}\right), 1,671(\mathrm{rC}=\mathrm{O}), 1,600(\mathrm{vC}=\mathrm{C}), 1,275$ (vCOC), UV ( $\mathrm{CH}_{3} \mathrm{OH}, \lambda$ in $\mathrm{nm}, \varepsilon$ in $\left.\mathrm{m}^{2} \cdot \mathrm{mol}^{-1}\right) ; \lambda_{\max } 206$ (3.23), 222 $\mathrm{nm}\left(\log \varepsilon_{1} 3.26\right), 275\left(\log \varepsilon_{2} 3.23\right)$

${ }^{1} \mathrm{H}-\mathrm{NMR}\left(\mathrm{CDCl}_{3}\right): 0.86-0.92\left(\mathrm{t}, 3 \mathrm{H}, \mathrm{CH}_{3 \text { alk }}{ }^{9}\right), 1.18-1.21(\mathrm{t}, 3 \mathrm{H}$, $\left.\mathrm{COCH}_{2} \mathrm{CH}_{3}\right), 1.24-1.27\left(\mathrm{~m}, 12 \mathrm{H}, \mathrm{CH}_{2}{ }_{\text {alk }}^{3-8}\right), 1.57-1.64\left(\mathrm{~m}, 2 \mathrm{H}, \mathrm{CH}_{2}\right.$ alk $\left.{ }^{2}\right)$, 2.92-3.00 (m, 2H, $\left.\mathrm{COCH}_{2} \mathrm{CH}_{3}\right), 3.51-3.55\left(\mathrm{t}, 2 \mathrm{H}, \mathrm{CH}_{2 \text { alk }}{ }^{1}\right)$, $4.74\left(\mathrm{~s}, 2 \mathrm{H}, \mathrm{Ar}-\mathrm{CH}_{2} \mathrm{O}\right), 6.81-6.86\left(\mathrm{~d}, 1 \mathrm{H}, \mathrm{CH}_{\mathrm{AR}}{ }^{6}\right), 7.79-7.83(\mathrm{~d}, 1 \mathrm{H}$, $\left.\mathrm{CH}_{\mathrm{AR}}{ }^{5}\right), 7.84-7.86\left(\mathrm{dd}, 1 \mathrm{H}, \mathrm{CH}_{\mathrm{AR}}{ }^{5}\right), 8.38(\mathrm{~s}, 1 \mathrm{H}, \mathrm{ArOH})$

${ }^{13} \mathrm{C}-\mathrm{NMR}\left(\mathrm{CDCl}_{3}\right)$ : $9.10\left(\mathrm{COCH}_{2} \mathrm{CH}_{3}\right), 14.61\left(\mathrm{C}_{\mathrm{alk}}{ }^{9}\right), 23.88\left(\mathrm{C}_{\mathrm{alk}}{ }^{8}\right)$, $27.45\left(\mathrm{C}_{\mathrm{alk}}{ }^{7}\right), 30.57\left(\mathrm{C}_{\mathrm{alk}}{ }^{6}\right), 30.72\left(\mathrm{C}_{\mathrm{alk}}{ }^{5}\right), 30.87\left(\mathrm{C}_{\mathrm{alk}}{ }^{4}\right), 30.89\left(\mathrm{C}_{\mathrm{alk}}{ }^{3}\right)$, $32.29\left(\mathrm{C}_{\text {alk }}{ }^{2}\right), 33.20\left(\mathrm{COCH}_{2} \mathrm{CH}_{3}\right), 68.75\left(\mathrm{Ar}-\mathrm{CH}_{2} \mathrm{O}\right), 71.93\left(\mathrm{C}_{\text {alk }}{ }^{1}\right)$, $115.89\left(C_{A R}{ }^{6}\right), 126.40\left(C_{A R}{ }^{2}\right), 129.85\left(C_{A R}{ }^{5}\right), 130.81\left(C_{A R}{ }^{4}\right), 131.03$ $\left(C_{A R}^{3}\right), 161.50\left(C_{A R}^{1}\right), 202.12(C O)$

\section{EP10n 1-(3-decyloxymethyl-4-hydroxyphenyl)propan-1-} one (13)

$\mathrm{C}_{20} \mathrm{H}_{32} \mathrm{O}_{3} \mathrm{M}_{\mathrm{r}} 320.48$, Anal. calcd. \%C $74.96 \% \mathrm{H} 10.06$ found $\% \mathrm{C}$ $74.80 \% \mathrm{H}$ 9.90. Yield: $62 \%, \mathrm{R}_{\mathrm{F}} 0.62, \mathrm{Mp} .52-54^{\circ} \mathrm{C}$ (heptane), IR $\left(\mathrm{cm}^{-1}\right), 3,343\left(\mathrm{rOH}_{\text {asoc. }}\right), 1,670(\mathrm{vC}=\mathrm{O}), 1,602(\mathrm{vC}=\mathrm{C}), 1,272$ (vCOC), UV $\left(\mathrm{CH}_{3} \mathrm{OH}, \lambda\right.$ in $\mathrm{nm}, \varepsilon$ in $\left.\mathrm{m}^{2} \cdot \mathrm{mol}^{-1}\right) ; \lambda_{\max } 222$ (log $\varepsilon_{1}$ 3.26), $275\left(\log \varepsilon_{2} 3.23\right)$

${ }^{1} \mathrm{H}-\mathrm{NMR}\left(\mathrm{CDCl}_{3}\right): 0.86-0.91\left(\mathrm{t}, 3 \mathrm{H}, \mathrm{CH}_{3 \text { alk }}{ }^{10}\right), 1.13-1.18(\mathrm{t}, 3 \mathrm{H}$, $\left.\mathrm{COCH}_{2} \mathrm{CH}_{3}\right), 1.26-1.40\left(\mathrm{~m}, 12 \mathrm{H}, \mathrm{CH}_{2}{ }_{\text {alk }}^{3-9}\right), 1.57-1.64(\mathrm{~m}, 2 \mathrm{H}$, $\left.\mathrm{CH}_{\text {2alk }}{ }^{2}\right), 2.88-3.00\left(\mathrm{~m}, 2 \mathrm{H}, \mathrm{COCH}_{2} \mathrm{CH}_{3}\right), 3.51-3.55\left(\mathrm{t}, 2 \mathrm{H}, \mathrm{CH}_{2 \text { alk }}{ }^{1}\right)$, $4.54\left(\mathrm{~s}, 2 \mathrm{H}, \mathrm{Ar}-\mathrm{CH}_{2} \mathrm{O}\right), 6.83-6.87\left(\mathrm{~d}, 1 \mathrm{H}, \mathrm{CH}_{\mathrm{AR}}{ }^{6}\right), 7.69-7.70(\mathrm{~d}, 1 \mathrm{H}$, $\left.\mathrm{CH}_{\mathrm{AR}}{ }^{3}\right), 7.74-7.86\left(\mathrm{dd}, 1 \mathrm{H}, \mathrm{CH}_{\mathrm{AR}}{ }^{5}\right), 8.40(\mathrm{~s}, 1 \mathrm{H}, \mathrm{ArOH})$

${ }^{13} \mathrm{C}-\mathrm{NMR}\left(\mathrm{CDCl}_{3}\right)$ : $9.10\left(\mathrm{COCH}_{2} \mathrm{CH}_{3}\right), 14.62\left(\mathrm{C}_{\text {alk }}{ }^{10}\right) 23.89\left(\mathrm{C}_{\mathrm{alk}}{ }^{9}\right)$, $27.46\left(\mathrm{C}_{\mathrm{alk}}{ }^{8}\right), 30.62\left(\mathrm{C}_{\mathrm{alk}}{ }^{7}, 30.67\left(\mathrm{C}_{\mathrm{alk}}{ }^{6}\right), 30.81\left(\mathrm{C}_{\mathrm{alk}}{ }^{5}\right), 30.86\left(\mathrm{C}_{\mathrm{alk}}{ }^{4}\right)\right.$, $30.89\left(\mathrm{C}_{\text {alk }}{ }^{3}\right), 32.29\left(\mathrm{C}_{\text {alk }}{ }^{2}\right), 33.22\left(\mathrm{COCH}_{2} \mathrm{CH}_{3}\right), 68.74\left(\mathrm{Ar}-\mathrm{CH}_{2} \mathrm{O}\right)$, $71.93\left(C_{\mathrm{alk}}{ }^{1}\right), 115.88\left(\mathrm{C}_{\mathrm{AR}}{ }^{6}\right), 126.40\left(\mathrm{C}_{\mathrm{AR}}{ }^{2}\right), 129.84\left(\mathrm{C}_{\mathrm{AR}}{ }^{5}\right), 130.80$ $\left(C_{A R}{ }^{4}\right), 131.02\left(C_{A R}^{3}\right), 161.49\left(C_{A R}{ }^{1}\right), 202.17(C O)$

\section{EP5C 1-(4-hydroxy-3-cyclopentyloxymethylphenyl) propan-1-one (14)}

$\mathrm{C}_{15} \mathrm{H}_{20} \mathrm{O}_{3} \mathrm{M}_{\mathrm{r}} 248.32$, Anal. calcd. \%C $72.55 \% \mathrm{H} 8.12$ found $\% \mathrm{C}$ 72.30, \%H 8.30, Yield: $62 \%, \mathrm{R}_{\mathrm{F}} 0.62 \mathrm{Mp} .68-69^{\circ} \mathrm{C}$ (heptane), IR $\left(\mathrm{cm}^{-1}\right), 3,255\left(\mathrm{rOH}_{\text {asoc. }}\right), 1,657(\mathrm{vC}=\mathrm{O}), 1,601 \quad(\mathrm{rC}=\mathrm{C}), 1,250$ (vCOC). UV $\left(\mathrm{CH}_{3} \mathrm{OH}, \lambda\right.$ in nm, $\varepsilon$ in $\left.\mathrm{m}^{2} \cdot \mathrm{mol}^{-1}\right) ; \lambda_{\max } 203$ (log $\varepsilon_{1}$ 3.17), $\lambda_{\max } 222\left(\log \varepsilon_{1} 3.20\right), 274\left(\log \varepsilon_{3} 3.19\right)$

${ }^{1} \mathrm{H}-\mathrm{NMR}\left(\mathrm{CDCl}_{3}\right): 1.19-1.22\left(\mathrm{t}, 3 \mathrm{H}, \mathrm{COCH}_{2} \mathrm{CH}_{3}\right), 1.59 ; 1.74-1.79$ $\left(\mathrm{m}, \mathrm{m}, 2 \mathrm{H}, 6 \mathrm{H}, \mathrm{CH}_{2}{ }^{\mathrm{ak} 2,3,4,5}\right), 2.91-2.96\left(\mathrm{q}, 2 \mathrm{H}, \mathrm{COCH}_{2} \mathrm{CH}_{3}\right), 4.08$ $\left(\mathrm{m}, 1 \mathrm{H}, \mathrm{CH}^{\mathrm{alk} 1}\right), 4.73\left(\mathrm{~s}, 2 \mathrm{H}, \mathrm{Ar}-\mathrm{CH}_{2} \mathrm{O}\right), 6.89-6.91\left(\mathrm{~d}, 1 \mathrm{H}, \mathrm{CH}_{\mathrm{AR}}{ }^{6}\right)$, 7.69-7.70 (d, 1 $\left.\mathrm{H} \mathrm{CH}_{\mathrm{AR}}{ }^{3}\right), 7.82-7.85\left(\mathrm{dd}, 1 \mathrm{H}, \mathrm{CH}_{\mathrm{AR}}{ }^{5}\right), 8.54(\mathrm{~s}, 1 \mathrm{H}$, $\mathrm{Ar}-\mathrm{OH})$

${ }^{13} \mathrm{C}-\mathrm{NMR} \quad\left(\mathrm{CDCl}_{3}\right): 9.09\left(\mathrm{COCH}_{2} \mathrm{CH}_{3}\right), 24.66\left(\mathrm{C}_{\mathrm{alk}}^{3,4}\right), 32.28$ $\left(\mathrm{COCH}_{2} \mathrm{CH}_{3}\right), 33.37\left(\mathrm{Calk}^{2,5}\right), 66.82\left(\mathrm{Ar}-\mathrm{CH}_{2} \mathrm{O}\right), 82.96\left(\mathrm{C}_{\mathrm{alk}}{ }^{1}\right)$, $115.82\left(C_{A R}^{6}\right), 126.70\left(C_{A R}{ }^{2}\right), 129.87\left(C_{A R}{ }^{5}\right), 130.77\left(C_{A R}{ }^{4}\right), 131.03$ $\left(C A R^{3}\right), 161.47\left(C_{A R}{ }^{1}\right), 202.23(C O)$ 
EPbenzyl 1-(3-phenylmethoxymethyl-4-hydroxyphenyl) propan-1-one (15)

$\mathrm{C}_{17} \mathrm{H}_{18} \mathrm{O}_{3} \mathrm{M}_{\mathrm{r}} 270$, Anal. calcd. \%C 75.53\% $\%$ 6.71 found \% C 75.40, $\% \mathrm{H}$ 6.50. Yield: $62 \%, \mathrm{R}_{\mathrm{F}} 0.62, \mathrm{Mp} .52-54^{\circ} \mathrm{C}$ (heptane), IR $\left(\mathrm{cm}^{-1}\right)$, 3,361 ( $\left.\mathrm{vOH}_{\text {asoc }}\right), 1,667(\mathrm{vC}=\mathrm{O}), 1,593(\mathrm{vC}=\mathrm{C}), 1,278$ ( $\left.\mathrm{vCOC}\right) . \mathrm{UV}$ $\left(\mathrm{CH}_{3} \mathrm{OH}, \lambda\right.$ in $\mathrm{nm}, \varepsilon$ in $\left.\mathrm{m}^{2} \cdot \mathrm{mol}^{-1}\right) ; \lambda_{\max } 206(\log \varepsilon, 3.39), \lambda_{\max } 219$ ( $\left.\log \varepsilon_{2} 3.29\right), 274\left(\log \varepsilon_{3} 3.19\right)$

${ }^{1} \mathrm{H}-\mathrm{NMR}\left(\mathrm{CDCl}_{3}\right):$ 1.19-1.22 (t, 3H, $\left.\mathrm{COCH}_{2} \mathrm{CH}_{3}\right), 2.90-2.96$ (q, $\left.2 \mathrm{H}, \mathrm{COCH}_{2} \mathrm{CH}_{3}\right), 4.62\left(\mathrm{~s}, 2 \mathrm{H}, \mathrm{CH}_{2}-\right.$ phenyl), $4.79\left(\mathrm{~s}, 2 \mathrm{H}, \mathrm{Ar}-\mathrm{CH}_{2} \mathrm{O}\right)$, 6.92-6.94 (d, 1H, $\left.\mathrm{CH}_{\mathrm{AR}}{ }^{6}\right), 7.33-7.40(\mathrm{~m}, 5 \mathrm{H}$, phenyl), 7.70-7.71 $\left(\mathrm{d}, 1 \mathrm{H}, \mathrm{CH}_{A R}{ }^{3}\right), 7.85-7.87\left(\mathrm{dd}, 1 \mathrm{H}, \mathrm{CH}_{\mathrm{AR}}{ }^{5}\right), 8.16(\mathrm{~s}, 1 \mathrm{H}, \mathrm{Ar}-\mathrm{OH})$

${ }^{13} \mathrm{C}-\mathrm{NMR}\left(\mathrm{CDCl}_{3}\right): 8.94\left(\mathrm{COCH}_{2} \mathrm{CH}_{3}\right), 32.13\left(\mathrm{COCH}_{2} \mathrm{CH}_{3}\right), 68.11$ $\left(\mathrm{Ar}-\mathrm{CH}_{2} \mathrm{O}\right), 73.60\left(\mathrm{CH}_{2}\right.$-phenyl), $115.75\left(\mathrm{C}_{\mathrm{AR}}{ }^{6}\right), 126.07\left(\mathrm{C}_{\text {phenyl }}{ }^{4}\right)$, $128.72\left(C_{A R}{ }^{2}\right), 128.96\left(C_{\text {phenyl }}^{2,6}\right), 129.39\left(C_{\text {phenyl }}^{3,5}\right), 129.73\left(C_{A R}{ }^{5}\right)$, $130.78\left(C_{A R}^{4}\right), 131.01\left(C_{A R}^{3}\right), 139.55\left(C_{\text {phenyl }}^{1}\right), 161.36\left(C_{A R}{ }^{1}\right)$, $202.06(C O)$

\section{ANTIMICROBIAL ACTIVITY}

Antimicrobial activity of prepared (3-alkoxymethyl-4hydroxyphenyl)propan-1-ones was evaluated in vitro and expressed as the minimum inhibitory concentration (MIC). It was determined using the standard broth dilution method (Valentová et al., 2018). The following strains of grampositive, gram-negative bacteria and a yeast pathogen were selected for the experiments: Staphylococcus aureus CNCTC Mau 29/58, Escherichia coli CNCTC 377/79 and Candida albicans CCM 8186, respectively. Tested bacterial strains were purchased from Czech National Collection of Type Cultures (Prague, Czech Republic); yeast was obtained from Czech Collection of Microorganisms (Brno, Czech Republic). For the sake of comparison, antibacterial activity of the antibiotic ciprofloxacin (1-cyclopropyl-6-fluoro-4-oxo-7-piperazin-1ylquinoline-3-carboxylic acid) was also evaluated.

\section{ANTIOXIDATIVE ACTIVITY}

\section{DPPH assay (Brand-Williams et al., 1995)}

The evaluation of antioxidative capacity by this method is based on the redox reaction of the tested compounds with the stable radical 2,2-diphenyl-1-(2,4,6-trinitrophenyl) hydrazyl (DPPH). The solution of this radical is purple coloured with maximum absorption at $517 \mathrm{~nm}$. In the course of the reduction of DPPH, the solution changes its colour from purple to yellow, resulting in corresponding shift in UV-VIS spectrum. The lower the measured absorption, the higher the antioxidative capacity of the tested compound.

A solution of DPPH in methanol was prepared, in the concentration $44 \mu \mathrm{g} / \mathrm{ml}(112 \mu \mathrm{M})$. Subsequently, solution of the tested sample in methanol in the concentration $10^{-2} \mathrm{~mol}$. $\mathrm{dm}^{-3}$ or $10^{-3} \mathrm{~mol} \mathrm{dm}^{-3}$ was prepared. For the spectrophotometric assay, $270 \mathrm{~mL}$ of the DPPH solution and $30 \mathrm{~mL}$ of tested compound solution or standard were mixed, and the absorbance using a microplate reader was determined at $517 \mathrm{~nm}$ at $5 \mathrm{~min}$ after mixing. The absorbance at each time point was corrected for the absorbance of a DPPH blank. Three parallel measurements were made for each sample. Trolox was used as a standard for measured antioxidant activity of the target compounds.

\section{ABTS assay (Re et al., 1999)}

Antiradical activity was measured as $\%$ inhibition of $\mathrm{ABTS}^{+}$. Aqueous solutions of ABTS $(7.7 \mu \mathrm{g} / \mathrm{ml}, 14 \mathrm{mM})$ and $\mathrm{K}_{2} \mathrm{~S}_{2} \mathrm{O}_{8}$ $(1.32 \mathrm{mg} / \mathrm{ml}, 4.9 \mathrm{mM})$ were prepared. These two solutions were mixed in a 1:1 vol. ratio and allowed to stand for $24 \mathrm{~h}$ in the refrigerator. The spectrophotometric measurement was carried out using a 96-well plate reader. Each well on the microplate was filled with $60 \mu$ of sample solution $\left(10^{-2}\right.$ or $10^{-3} \mathrm{~mol}_{\mathrm{dm}} \mathrm{d}^{-3}$, respectively), and $240 \mu \mathrm{l}$ of ABTS solution. Absorbance was assessed spectrophotometrically at 734 $\mathrm{nm}$ at $5 \mathrm{~min}$ after mixing. For each sample, three parallel measurements were made. During the reaction, the colourless 2,2'-azino-bis(3-ethylbenzothiazoline-6-sulfonic acid is oxidised by potassium peroxydisulfate, yielding the stable blue-green ABTS. ${ }^{+}$radical. The addition of antioxidants leads to reduction of the ABTS.+ radical and discolouration of the solution.

\section{DISCUSSION}

The aim of the presented research was the synthesis of (3-alkoxymethyl-4-hydroxyphenyl)propan-1-ones (Table 1), screening of antimicrobial and antioxidant activities of selected products, as well as comparison of the results with the previously prepared (3-alkoxymethyl-4-hydroxyphenyl) ethanones and (2RS)-bis [3-(4-acetyl-2-propoxymethyl) phenoxy-2-hydroxypropyl]isopropylammonium fumarate with beta-blocking activity.

The compounds 1-15 (Table 1) were synthesised by an establishedtwo-step procedurefrom4-hydroxyphenylpropan1-one (Čižmáriková et al., 1991). During the first stage, (3-chloromethyl-4-hydroxyphenyl)propan-1-one was prepared in $57 \%$ yield via electrophilic substitution reaction. This intermediate reacts in the second step of the synthesis with the respective alcohol in the presence of $\mathrm{NaHCO}_{3}$ to give (3-alkoxymethyl-4-hydroxyphenyl)propan-1-ones in $60 \%$ yield. The products are white solids with $\mathrm{mp}$ between 30 and $70^{\circ} \mathrm{C}$. Their purity was checked by TLC on silica, the mobile phase consisting of cyclohexane/ethyl acetate in $8: 2 \mathrm{v} / \mathrm{v}$ ratio. The structure of the final (3-alkoxymethyl-4-hydroxyphenyl) propan-1-ones 1-15 was confirmed by spectral analysis. The following bands could be assigned in the infrared spectra: $3,236-3,352 \mathrm{~cm}^{-1}\left(\mathrm{rOH}_{\text {asoc }}\right), 1,653-1,686 \mathrm{~cm}^{-1}(\mathrm{vC}=0), 1,596-$ $1,604 \mathrm{~cm}^{-1}(\mathrm{vC}=\mathrm{C})$ and $1,270-1,275 \mathrm{~cm}^{-1}$ (vCOC). In the ${ }^{1} \mathrm{H}$ and ${ }^{13} \mathrm{C}-\mathrm{NMR}$ spectra, the signals of the aromatic ring, the propanoyl and the alkoxymethyl groups were identified. Two or four bands can be seen in the UV spectra, corresponding to $\pi-\pi^{*}$ transitions at 202-206, 216-227, 264 and 274-277 nm. 
Čižmáriková $R$. et al.

Table 2. Antimicrobial activity of 1-[3-(alkoxymethyl)-4-hydroxyphenyl]alkanones.

\begin{tabular}{|c|c|c|c|}
\hline Substance Number & Description & MIC (mmol/L) E.coli & MIC (mmol/L) S. aureus \\
\hline 2 & EP2 & 5.01 & 5.01 \\
\hline 3 & EP3n & 2.46 & $\mathrm{n}$ \\
\hline 6 & EP4i & 1.06 & $\mathrm{n}$ \\
\hline 9 & EP6n & 0.38 & 1.43 \\
\hline 12 & EP9n & 1.43 & 0.13 \\
\hline 13 & EP10n & 0.77 & 0.65 \\
\hline 14 & EP5C & 1.29 & 0.31 \\
\hline 15 & EPbenz & 1.25 & $\mathrm{n}$ \\
\hline $1 a$ & $40 H P r$ & $\mathrm{n}$ & $\mathrm{n}$ \\
\hline 18 & EA4n & 0.31 & $\mathrm{n}$ \\
\hline 20 & EA7n & 0.94 & $\mathrm{n}$ \\
\hline 21 & EA8n & 0.09 & 2.05 \\
\hline 28 & EA5c & 4.11 & $\mathrm{n}$ \\
\hline
\end{tabular}

$n=$ non-measurable values, compounds not active against Candida albicans

Table 3. Antioxidant activities of 1-[3-(alkoxymethyl)-4-hydroxyphenyl]alkanones.

\begin{tabular}{|c|c|c|c|}
\hline Substance Number & Description & Inhibition DPPH [\%]土SD & Inhibition ABTS [\%] \pm SD \\
\hline 1 & EP1 & $\mathrm{n}$ & $3.9 \pm 0.04$ \\
\hline 2 & EP2 & $2.5 \pm 0.1$ & $8.5 \pm 0.4$ \\
\hline 3 & EP3n & $0.6 \pm 0.01$ & $11.9 \pm 0.4$ \\
\hline 4 & EP3i & $3.7 \pm 0.02$ & $28.1 \pm 0.3$ \\
\hline 6 & EP4i & $2.4 \pm 0.7$ & $10.2 \pm 0.2$ \\
\hline 7 & EP5n & $1.8 \pm 1.3$ & $1.3 \pm 0.4^{*}$ \\
\hline 8 & EP5i & $12.6 \pm 0.6$ & $5.8 \pm 0.7^{*}$ \\
\hline 11 & EP8n & $6.2 \pm 0.7$ & $\mathrm{n}$ \\
\hline 13 & EP10n & $6.3 \pm 2.1$ & $\mathrm{n}$ \\
\hline 14 & EP5C & $3.3 \pm 0.03$ & $25.0 \pm 1.6$ \\
\hline 15 & EPbenzyl & $3.3 \pm 0.9$ & $1.5 \pm 0.3^{*}$ \\
\hline 16 & EA1 & $n$ & $6.1 \pm 0.2$ \\
\hline 17 & EA2 & $\mathrm{n}$ & $1.4 \pm 1.0$ \\
\hline 18 & EA3n & $\mathrm{n}$ & $6.1 \pm 0.7$ \\
\hline 19 & EA3i & $\mathrm{n}$ & $7.5 \pm 0.2$ \\
\hline 20 & EA4n & $\mathrm{n}$ & $1.5 \pm 1.0$ \\
\hline 21 & EA5n & $3.9 \pm 0.8$ & $24.8 \pm 0.8$ \\
\hline 22 & EA7n & $\mathrm{n}$ & $0.9 \pm 0.2^{*}$ \\
\hline 23 & EA8n & $\mathrm{n}$ & $\mathrm{n}$ \\
\hline 24 & EA9n & $3.9 \pm 0.7$ & $\mathrm{n}$ \\
\hline 25 & EA5c & $\mathrm{n}$ & $8.4 \pm 0.7$ \\
\hline 26 & EAbenzyl & $1.59 \pm 1.4$ & $16.7 \pm 0.7$ \\
\hline 27 & 4-OHacet & $\mathrm{n}$ & $0.9 \pm 0.01$ \\
\hline 28 & EAch & $4.32 \pm 2.5$ & $8.2 \pm 0.4^{*}$ \\
\hline
\end{tabular}

"concentration $10^{-3} \mathrm{~mol} . \mathrm{dm}^{-3} \mathrm{n}=$ non-measurable values 


\section{Synthesis, antimicrobial and antiradical activity of (3-alkoxymethyl-4-hydroxyphenyl)propan-1 ...}<smiles>[R]Cc1ccc(OCC(O)C[NH3+])c(C(C)C)c1</smiles>

Table 4. Antioxidative activity of (2RS)-bis [3-(4-acetyl-2-alkoxymethyl)phenoxy-2-hydroxypropyl]isopropylammonium fumarate exhibiting beta-blocking activity.

\begin{tabular}{|c|c|c|c|c|}
\hline Description & $\mathbf{R 1}$ & $\mathbf{R 2}$ & Inhibition DPPH [\%] \pm SD & Inhibition ABTS [\%] \pm SD \\
\hline FA23i & $\mathrm{CH}_{3}$ & $\mathrm{CH}_{2} \mathrm{CH}_{3}$ & $\mathrm{~N}$ & $99.0 \pm 1.3$ \\
\hline FA5n3i & $\mathrm{CH}_{3}$ & $\left(\mathrm{CH}_{2}\right)_{4} \mathrm{CH}_{3}$ & $\mathrm{~N}$ & $48.5 \pm 0.8$ \\
\hline $\mathrm{FA5i3i}$ & $\mathrm{CH}_{3}$ & $\mathrm{CH}_{2} \mathrm{CH}_{2} \mathrm{CH}\left(\mathrm{CH}_{3}\right)_{2}$ & $0.4 \pm 0.04$ & $57.2 \pm 1.4$ \\
\hline FA7n3i & $\mathrm{CH}_{3}$ & $\left(\mathrm{CH}_{2}\right)_{6} \mathrm{CH}_{3}$ & $\mathrm{~N}$ & $89.2 \pm 1.3$ \\
\hline FA8n3i & $\mathrm{CH}_{3}$ & $\left(\mathrm{CH}_{2}\right)_{7} \mathrm{CH}_{3}$ & $\mathrm{~N}$ & $86.7 \pm 5.3$ \\
\hline FA9n3i & $\mathrm{CH}_{3}$ & $\left(\mathrm{CH}_{2}\right)_{8} \mathrm{CH}_{3}$ & $1.6 \pm 0.04$ & $\mathrm{n}$ \\
\hline FAB3i & $\mathrm{CH}_{3}$ & $\mathrm{CH}_{2}$ phenyl & $4.6 \pm 1.9$ & $97.5 \pm 2.3$ \\
\hline Propranolol & & & \\
\hline
\end{tabular}

$n=$ non-measurable values<smiles>CC(C)NCC(O)COc1cccc2ccccc12</smiles>

Table 5. Antimicrobial activity of (2RS)- bis [3-(4-propionyl-2-alkoxymethyl)phenoxy-2-hydroxypropyl]isopropylammonium fumarate.

\begin{tabular}{|c|c|c|c|c|c|}
\hline Description & $\mathbf{R} 1$ & $\mathbf{R} 2$ & $\begin{array}{c}\mathbf{M I C}(\mathbf{m m o l} / \mathbf{L}) \\
\text { E. coli }\end{array}$ & $\begin{array}{c}\text { MIC(mmol/L) } \\
\text { S. aureus }\end{array}$ & $\begin{array}{c}\text { MIC(mmol/L) } \\
\text { C. albicans }\end{array}$ \\
\hline FpP4n3i & $\mathrm{CH}_{3} \mathrm{CH}_{2}$ & $\left(\mathrm{CH}_{2}\right)_{3} \mathrm{CH}_{3}$ & $\mathrm{~N}$ & 0.61 & $\mathrm{n}$ \\
\hline FpP5n3i & $\mathrm{CH}_{3} \mathrm{CH}_{2}$ & $\left(\mathrm{CH}_{2}\right)_{4} \mathrm{CH}_{3}$ & $\mathrm{~N}$ & 0.35 & $\mathrm{n}$ \\
\hline FpP6n3i & $\mathrm{CH}_{3} \mathrm{CH}_{2}$ & $\left(\mathrm{CH}_{2}\right)_{5} \mathrm{CH}_{3}$ & 0.34 & 0.08 & 0.23 \\
\hline FpP7n3i & $\mathrm{CH}_{3} \mathrm{CH}_{2}$ & $\left(\mathrm{CH}_{2}\right)_{6} \mathrm{CH}_{3}$ & 0.22 & 0.03 & 0.07 \\
\hline FpP9n3i & $\mathrm{CH}_{3} \mathrm{CH}_{2}$ & $\left(\mathrm{CH}_{2}\right)_{8} \mathrm{CH}_{3}$ & 0.20 & 0.01 & 0.01 \\
\hline FpA5n3i & $\mathrm{CH}_{3}$ & $\left(\mathrm{CH}_{2}\right)_{4} \mathrm{CH}_{3}$ & $\mathrm{~N}$ & 0.61 & $\mathrm{n}$ \\
\hline FpA6n3i & $\mathrm{CH}_{3}$ & $\left(\mathrm{CH}_{2}\right)_{5} \mathrm{CH}_{3}$ & 0.35 & 0.08 & 0.24 \\
\hline FpA7n3i & $\mathrm{CH}_{3}$ & $\left(\mathrm{CH}_{2}\right)_{7} \mathrm{CH}_{3}$ & 0.23 & 0.07 & 0.23 \\
\hline FpA8n3i & $\mathrm{CH}_{3}$ & $\left(\mathrm{CH}_{2}\right)_{7} \mathrm{CH}_{3}$ & 0.22 & 0.03 & 0.08 \\
\hline FpA9n3i & $\mathrm{CH}_{3}$ & $\left(\mathrm{CH}_{2}\right)_{8} \mathrm{CH}_{3}$ & 0.21 & 0.01 & 0.02 \\
\hline ciprofloxacin & & & $3.10-4$ & $6.10^{-4}$ & $\mathrm{n}$ \\
\hline
\end{tabular}

$n=$ non-measurable values 
The compounds 16-26 (Table 1) derived from 4-hydroxyphenylethanones are described in (Čižmáriková et al., 2002).

Antimicrobial activity of selected final products (Table 2) was tested against gram-negative bacterium (Escherichia coli), gram-positive bacterium (Staphylococcus aureus) and human fungal pathogen (Candida albicans).

The comparison between the tested compounds showed that maximum activity against $E$. coli can be found in the propanone derivative with the hexyloxymethyl-side chain (EP6n, MIC $0.38 \mathrm{mmol} / \mathrm{L})$. Ethanone derivative with the butoxymethyl group (EA4n, MIC $0.31 \mathrm{mmol} / \mathrm{L}$ ) showed similar activity. Comparison of activities of ethanone (EA5c) and propanone (EP5c) derivatives with the cyclopentyloxymethyl moiety revealed that the activity of the propanone derivative was higher (MIC $1.29 \mathrm{mmol} / \mathrm{L}$ ) than the activity of the ethanone derivative (MIC $4.11 \mathrm{mmol} / \mathrm{L}$ ). Prolongation of the alkyl chain caused an increase in activity from ethoxymethyl up to nonyloxymethyl (EP2 through EP9n).

Similar observation was made also with $S$. aureus. The highest effect was shown by the compound with decyloxymethyl substituent (EP10n, MIC $0.13 \mathrm{mmol} / \mathrm{L})$. The compounds EP2 and EP10n exerted comparable activity both in E. coli and $S$. aureus. None of the tested compounds exhibited substantial activity against $C$. albicans.

Published data suggest that compounds with one or several phenolic hydroxyls act as radical scavengers and exert antioxidative activity. Hence, they impede oxidative stress, a condition that can be the main cause of numerous diseases, especially those of the cardiovascular system. The antioxidative activities of the prepared compounds were evaluated using methods based on DPPH (1,1-diphenyl-2-picrylhydrazyl) and ABTS $^{+}$(2,2'-azinobis(3-ethylbenzothiazoline-6-sulfonic acid). The DPPH method involves the reaction between the antioxidant and the stable radical DPPH•, which acts as acceptor of hydrogen. The solution of this radical has intensely purple hue, caused by an unpaired electron of the hydrazyl group. Its reaction with the antioxidant yields the reduced form DPPH-H, and the solution discolours in the course of this reaction. The degree of antioxidant activity is determined from the decrease of absorbency of the solution at $517 \mathrm{~nm}$ wavelength. The ABTS method employs oxidation of the colourless 2,2 azinobis(3-ethylbenzothiazoline-6-sulfonic acid) by potassium peroxydisulfate, yielding the stable bluegreen radical-cation $\mathrm{ABTS} .^{+}$. The addition of antioxidants to such a solution leads to reduction of the ABTS. ${ }^{+}$radical and discolouration of the solution.

The antioxidant activity of Trolox (6-hydroxy-2,5,7,8tetramethylchroman-2-carboxylic acid) used as standard was determined along with the activities of investigated products. The values of antioxidative activities (Table 3 ) determined by the DPPH method were generally lower than those provided by the ABTS methods, in some cases, even below the detection threshold. The antioxidative activities of the products were in the range $0.6-4.3 \%$, with the exception of the substance
EP5i, in which case, it was $12.6 \%$. The ABTS method provided activities in the range $0.9-28.1 \%$, the highest activity being found in the substance EP3i with isopropoxymethyl substituent (28.1\%). Neither of the methods provides a clear dependency between the activity and the length of the alkyl substituent.

The majority of the compounds in the group of 3-alkoxymethyl-4-hydroxyphenylethanones (16-26) did not show any activity detectable by the DPPH method. The highest activity provided by the ABTS method was found in the compound with pentyloxymethyl substituent (EA5n, 24.8\%).

Low ABTS value was shown also by the parent structure 4-hydroxyphenylethanone. 3-Chloromethyl-4hydroxyphenylethanones exerted higher activity both by the DPPH (4.4\%) and ABTS (8.2\%) methods (Table 3).

The activities correspond to the values for antioxidative activities of phenols reported in the literature. Compounds with only one hydroxy group in the molecule exhibit only minor antioxidant effects, markedly higher values being shown by compounds with two or more hydroxy groups (Sroka \& Cisowski, 2003).

Several previously reported compounds with betaadrenolytic effect (Čižmáriková et al., 1985; 1986; 2003) were tested for comparison. The compounds exerted marked anti-isoprenaline activity with negative chronotropic, dromotropic and inotropic effects. Optimum antiarrhythmic and anticonvulsive activity was found in derivatives with methoxymethyl and propoxymethyl groups. To assess the antioxidant activity, (2RS)-bis[3-(4-acetyl-2-propoxymethyl) phenoxy-2-hydroxypropyl]isopropylammonium fumarate with varying length of the alkoxymethyl chain was selected. The ABTS method was found to be more convenient compared to the DPPH assay. The antioxidative (Table 4) activities acquired by the ABTS method were in the range $52.2-99.0 \%$, the highest effect being observed in ethoxymethyl (FA23i, 99.0\%) and octyloxymethyl (FA8n3i, 89.8\%) derivatives (Table 4). Similarly to the investigated substances, the antioxidative activity of the standard compound propranolol appraised by the ABTS method (4.6\%) was higher in comparison with $\mathrm{DPPH}$.

In some cases (EP5, EPbenzyl, EA7, EAchlormet), the antioxidant activities were assessed at a lower concentration $\left(10^{-3} \quad \mathrm{~mol} . \mathrm{dm}^{-3}\right) . \quad$ (2RS)-bis[3-(4-acetyl-2-propoxymethyl) phenoxy-2-hydroxypropyl]isopropylammonium fumarate was more active than the phenolic derivatives, suggesting potentiation of antioxidative activity as a result of introduction of isopropylaminopropanol moiety into the molecule. The published results implicate correlation between the antioxidant activity, connected to reduction in oxidative stress and therapeutic effect in cardiovascular disease. The antimicrobial activity (Table 5) of selected (2RS)-bis[3(4-acetyl-2-propoxymethyl)phenoxy-2-hydroxypropyl] isopropylammonium fumarate in tested microbial strains was higher in comparison with the intermediate etanones and 
propanones. This increase in activity stems from the elevated lipophilicity connected to prolongation of the alkoxymethyl chain. In both series, the highest effect on all tested strains was observed in compounds with nonyloxymethyl substituent in the side chain. In comparison with the standard ciprofloxacin, the antimicrobial activities were inferior in both groups.

Considering both types of bioactivity, the antimicrobial effects (in S. aureus and E. coli) and the antioxidant activity (by
ABTS assay), were both higher in comparison with the (2RS)bis [3-(4-acetyl-2-propoxymethyl)phenoxy-2-hydroxypropyl] isopropylammonium fumarate with beta-blocking activity.

\section{ACKNOWLEDGEMENTS}

This work has been supported by the Scientific Grants Agency of the Slovak Republic (VEGA 1/0145/20).

\section{References}

[1] Abbaszadeh S, Sharifzadeh A, Shokri H, Khosravi AR, Abbaszadeh A. Antifungal efficacy of thymol, carvacrol, eugenol and menthol as alternative agents to control the growth of food-relevant fungi. J Mycol Med. 2014;24(2):e51-56.

[2] Amouar E, Calliste CA, Košinova P, Di Meo F, Duroux JL, Champavier $Y$. Free radical scavenging properties of guaiacol oligomers: A combined experimental and quantum study of the guaiacyl-moiety role. J Phys Chem A. 2009;113:13881-13891.

[3] Bendary E, Francis, RR, Ali HMG, Sarwat MI, El Hady S. Antioxidant activity and structure-activity relationships (SARs) of some phenolic and anilines compounds. Ann Agric Sci. 2013;58(2):173181.

[4] Brand-Williams W, Cuvelier ME, Berset C. Use of a free radical method to evaluate antioxidant activity. LWT-Food Sci Technol. 1995;28(1):25-30.

[5] Calliste CA, Le Bail JC, Trouillas P, Pouget C, Habrioux G, Chulia AJ, Duroux JL.

Chalcones: structural requirements for antioxidant, estrogenic and antiproliferative activities. Anticancer Res. 2001;21(6A):39493956.

[6] Čižmáriková R, Borovanský A, Béderová E, Kozlovský J, Dingová A, Zdráhalová Z. Derivatives of p-hydroxypropiophenone as potential beta-adrenolytics agents of the aryloxyaminopropanols type. Českoslov Farm. 1986;35:110-113.

[7] Čižmáriková R, Borovanský A, Čižmárik J, Hücklová M. 3-alkoxymetyl-4-hydroxyfenylalkylketóny a spôsob ich výroby. CS pat. 1991;269 565 .

[8] Čižmáriková R, Borovanský A, Kozlovský J, Béderová E, Dingová A. 4-(3-alkylamino-2-hydroxypropoxy(-3-(alkoxymethyl) acetophenones. Collect Czech Chem Commun. 1985;50:22892297.

[9] Čižmáriková R, Polakovičová M, Mišíková E. Synthesis, physicochemical properties and conformational studies of (3-alkoxymethyl-4-hydroxyphenyl)ethanones. Chem Pap. 2002;56:256-260.

[10] Čižmáriková R, Račanská E, Hroboňová K, Lehotay J, Mišíková E. Synthesis and enantioseparation of some arylaminopropanol derivatives and their effects on the central nervous system. Acta Facult Pharm Univ Comenianae. 2003;50:38-50.

[11] Cueva C, Moreno-Arribas MV, Martín-Álvarez PJ, Bills G, Vicente MF, Basilio A, Rivas $C L$, Requena $T$, Rodríguez JM, Bartolomé B. Antimicrobial activity of phenolic acids against commensal, probiotic and pathogenic bacteria. Res Microbiol. 2010;161(5):372-382.
[12] da Re P, Verlichi L. Chloromethylation of hydroxypropiophenone and synthesis of alcohols and aldehydes of chromone group. Ann Chim. 1956;46:910-914. Chem. Abstr. 51c 6618f.

[13] Devi KP, Nisha SA, Sakthivel R, Pandian SK. Eugenol (an essential oil of clove) acts as an antibacterial agent against Salmonella typhi by disrupting the cellular membrane. J Ethnopharmacol. 2010;6;130(1):107-115.

[14] Fernandez MT, Mira ML, Florencio MH, Jennings KR. Iron and copper chelation by flavonoids: an electrospray mass spectrometry study. J Inorg Biochem. 2002;92(2):105-111.

[15] Fujisawa S, Atsumi T, Kadoma Y, Ishihara M, Ito S, Yokoe I. Kinetic radical scavenging activity and cytotoxicity of 2-methoxy- and 2-t-butyl-substituted phenols and their dimers. Anticancer Res. 2004; 24(5A):3019-3026.

[16] Fujisawa S, Atsumi T, Kadoma Y, Sakagami H. Antioxidant and prooxidant action of eugenol-related compounds and their cytotoxicity. Toxicology. 2002;177(1):39-54.

[17] Hider RC, Liu ZD, Khodr HH. Metal chelation of polyphenols. Methods Enzymol. 2001;335:190-203.

[18] Irie Y, Itokazu N, Anjiki N, Ishige A, Watanabe K, Keung WM. Eugenol exhibits antidepressant-like activity in mice and induces expression of metallothionein-III in the hippocampus. Brain Res. 2004;1011(2):243-246.

[19] Kadoma Y, Ito S, Atsumi T, Fujisawa S. Mechanism of cytotoxicity of 2- or 2,6-terc-butylphenols and 2-methoxyphenols in terms of inhibition rate constant and theoretical parameter. Chemosphere. 2009;74:626-632.

[20] Kadoma Y, Ito S, Yokoe I, Fujisawa S. Comparative study of the alkyl and peroxy radical-scavenging activity of 2-t-butyl4-methoxyphenol (BHA) and its dimer, and their theoretical parameters. In Vivo. 2008; 22(3):289-296.

[21] Kadoma Y, Murakami Y, Ogiwara T, Machino M, Yokoe I, Fujisawa S. Radical-scavenging activity and cytotoxicity of p-methoxyphenol and $p$-cresol dimers. Molecules. 2010; 26;15(3):1103-1112.

[22] Morcia C, Malnati M, Terzi V. In vitro antifungal activity of terpinen4-ol, eugenol, carvone, 1,8-cineole (eucalyptol) and thymol against mycotoxigenic plant pathogens. Food Addit Contam Part A Chem Anal Control Expo Risk Assess. 2012;29(3):415-422.

[23] Park E, Moon W, Song, M, Kim M, Chung K, Yoon J. Antimicrobial activity of phenol and benzoic acid derivatives. Int Biodeterior Biodegradation. 2001;47(4):209-214.

[24] Re R, Pellegrini N, Proteggente A, Pannala A, Yang M, Rice-Evans C. Antioxidant activity applying an improved ABTS radical cation decolorization assay. Free Radic Biol Med. 1999;26(9):1231-1237. 
[25] Sroka Z, Cisowski W. Hydrogen peroxide scavenging, antioxidant and anti-radical activity of some phenolic acids. Food Chem Toxicol. 2003;41(6):753-758.

[26] Taguri T, Tanaka T, Kouno I. Antibacterial spectrum of plant polyphenols and extracts depending upon hydroxyphenyl structure. Biol Pharm Bull. 2006;29(11):2226-2235.

[27] Taguri T, Tanaka T, Kouno I. Antimicrobial activity of 10 different plant polyphenols against bacteria causing food-borne disease. Biol Pharm Bull. 2004;27(12):1965-1969.

[28] Valentová J, Varényi S, Herich P, Baran P, Bilková A, Kožíšek J, Habala L. Synthesis, structures and biological activity of copper(II) and zinc(II) Schiff base complexes derived from aminocyclohexane-1-carboxylic acid. New type of geometrical isomerism in polynuclear complexes. Inorganica Chim Acta. 2018;480:16-26. 